\title{
Influence of viscoelasticity on drop deformation and orientation in shear flow.
}

\section{Part 2: Dynamics}

K. Verhulst, R. Cardinaels, P. Moldenaers

Lab for Applied Rheology and Polymer Processing

Department of Chemical Engineering

KU Leuven

Willem de Croylaan 46, Box 2423, B-3001 Leuven, Belgium

Paula.Moldenaers@cit.kuleuven.be

S. Afkhami, Y. Renardy

Department of Mathematics and ICAM

Virginia Polytechnic Institute and State University

460 McBryde Hall, Blackburg, VA24061-0123, USA

Renardyy@aol.com

Final accepted draft

Cite as: K. Verhulst, R. Cardinaels, P. Moldenaers, S. Afkhami, Y. Renardy, J. Non-Newt. Fluid Mech., 156(1-2), pp. 44-57 (2009)

The original publication is available at:

http://www.sciencedirect.com/science/article/pii/S0377025708001778 


\title{
Influence of viscoelasticity on drop deformation and orientation in shear flow Part 2: Dynamics
}

\author{
Kristof Verhulst ${ }^{a}$ Ruth Cardinaels ${ }^{a}$ Paula Moldenaers ${ }^{\mathrm{a}}$ \\ ${ }^{a}$ Katholieke Universiteit Leuven, Department of Chemical Engineering, W. de \\ Croylaan 46 - B 3001 Heverlee (Leuven), Belgium \\ Shahriar Afkhami ${ }^{\text {b }}$ Yuriko Renardy ${ }^{\text {b }}$ \\ ${ }^{\mathrm{b}}$ Department of Mathematics and ICAM, 460 McBryde Hall, Virginia Polytechnic \\ Institute and State University, Blackburg VA 24061-0123, USA
}

\begin{abstract}
An experimental study of drop dynamics under shear is conducted for five fluid pairs: a reference Newtonian system, two systems with a viscoelastic drop in a Newtonian matrix, one with a Newtonian drop in a viscoelastic matrix, all at drop to matrix viscosity ratio $\lambda=1.5$, and a separate case at $\lambda=0.75$. The viscoelastic liquids are either a Boger fluid or a shear-thinning viscoelastic fluid satisfying an Ellis model. Deborah numbers in the range 1 to 2 and a range of capillary numbers from low to above breakup conditions are addressed. The results focus on three aspects: relaxation after cessation of shear, a new viscoelastic drop breakup scenario, and the effect of shear flow history on drop breakup. Numerical simulations with the 3D volume-of-fluid PROST method complement the experimental results.
\end{abstract}

Key words: drop deformation, drop breakup, Oldroyd-B, VOF method, blend morphology, viscoelasticity

\section{Introduction}

An immiscible polymer blend is typically a multi-phase material that displays a droplet-matrix morphology [1]. During the manufacturing process, an in-

1 Corresponding author:

Email address: renardyy@aol.com Y. Renardy

Preprint submitted to Elsevier $\quad 5$ August 2008 
dividual droplet at the microscopic level deforms to various shapes, breaks up or interacts with neighboring droplets, and experiences a complicated flow history [2]. Accurate prediction of how the morphology evolves in the mixer is thus essential in the development of polymer blends with specific material properties. In this paper, we address the dynamics of a single droplet during processing in the context of simple shear. The simplified model of a constant shear rate applied to a single droplet has attracted numerous theoretical and experimental studies, starting with the Newtonian-Newtonian systems $[3,4,5]$. For example, experimental data and 3D direct simulations for the transient deformation and orientation upon startup at viscosity ratio 1.5 agree quite well in figure 1 of [6]. The drop evolves monotonously toward the stationary state. Behavior at sufficiently low deformation is also captured by several phenomenological models; for example, the Maffettone-Minale model [7].

Recent investigations address viscoelastic components [8, 9, 10]. Matrix viscoelasticity is now known to significantly affect the droplet dynamics. First, severe overshoots may occur in the transient evolution toward a stationary state, either in startup of shear flow $[11,12,13]$ or startup of planar extensional flow [14]. Possible causes for this overshoot are discussed in [15]. Secondly, matrix viscoelasticity slows down the droplet shape relaxation after cessation of flow [14, 13], even at small deformations [16]. For small deformations, the experimental results agree qualitatively with phenomenological models $[16,17,18,13]$ although overshoots are generally underpredicted. Moreover, the experimentally observed relaxation process is substantially slower than the predicted one, especially during the final phase [16, 13]. Verhulst et al. [13] suggest that the simplification of a single relaxation mode contributes to these quantitative disagreements.

The effect of droplet viscoelasticity on transient behavior is investigated experimentally in $[12,19]$, which show less pronounced effects than the viscoelastic matrix case. The 2D numerical simulations of $[20,21]$ also support this conclusion. The reason is that when the matrix liquid is Newtonian, the imposed velocity field is felt by the viscoelastic medium in a limited way, away from high velocities. Hence, the droplet shape remains close to spherical, and the flow field inside the droplet is highly rotational $[6,15,20]$. Rotational flows induce small elastic stresses and viscoelastic effects then hardly affect the stationary-state deformation and orientation, even at higher capillary and Deborah numbers ( $c f$. numerical simulations in part 1 of this work [6]). When an overshoot does occur, Yue et al. [20] attribute it to a mismatch of two time scales: (i) the capillary time of drop deformation and (ii) the relaxation time of the polymers in the viscoelastic matrix phase. At high flow rates, the elastic stresses at both the droplet tip and equator grow more slowly than the droplet deforms. Hence, the initial transients reflect Newtonian behavior. Then, after a finite time, elastic stresses become large enough to suppress the deformation, resulting in the steady droplet shape. 
Droplet breakup in blends with at least one viscoelastic component is studied in the experimental investigations of $[22,23,24,25,26]$, which conclude that matrix and droplet viscoelasticity both inhibit droplet breakup. However, the majority of these studies include other hydrodynamic effects, nonhomogeneities in the applied flow field, shear-thinning behavior, or differences in the viscosity ratio among the various blend systems. A clearer distinction of the effect of component viscoelasticity is obtained in [11] for breakup of a single Newtonian droplet in a Boger fluid under simple shear. It is found that matrix viscoelasticity indeed inhibits droplet breakup in shear. It is also shown that matrix viscoelasticity increases the non-dimensional pinch-off length and time, although the breakup mechanism itself is similar to that observed for Newtonian-Newtonian systems. In planar extensional flow, on the other hand, matrix viscoelasticity enhances droplet deformation, thus decreasing the critical capillary number $\left(\mathrm{Ca}_{c r}\right)$ for breakup [14]. The critical pinch-off length remains constant.

The breakup of a viscoelastic drop in a Newtonian liquid for small elasticity is similar to the Newtonian-Newtonian systems, which display bulbous ends during end-pinching for viscosity ratio 1, or cusped droplet ends for low viscosity ratios [27]. On the other hand, significant differences can arise, depending on the physical properties of the droplet and matrix phase, and the rheology of the fluids. For instance, Migler [28] reports the alignment and breakup in the vorticity direction for highly elastic droplets under strong shear. A variety of systems, flow types, and experimental conditions give rise to this phenomenon $[27,29,30,31,32,33]$. It is generally accepted that alignment and breakup along the vorticity direction involve a complicated interplay between the first and second normal stress differences of both phases, the viscosity ratio, shearthinning behavior, and the applied capillary number. In addition, since the process takes a relatively long time, a comparison with direct numerical simulations is yet to be performed. In less severe flow conditions, there are threedimensional droplet breakup simulations on systems containing a viscoelastic phase $[9,34,35]$.

In this paper, the influence of component viscoelasticity on droplet dynamics is studied. Section 3 addresses shape relaxation after cessation of shear flow, which is related to the normal modes of oscillation of a spherical drop. This problem has been studied for limiting cases such as the linearized motion of a viscous drop in another viscous fluid [36], and a viscoelastic drop in vacuum[37]. Section 4 describes drop breakup in simple shear where either the drop or the matrix liquid is viscoelastic. In the case of the viscoelastic drop at viscosity ratio 1.5, close to critical conditions, a new two-step breakup procedure is found experimentally. Section 5 focusses on the effect of shear flow history on droplet breakup. For Stokes flow of viscous liquids, the elliptic nature of the governing equations ensures that a drop evolves to a stationary state or to breakup, independent of the initial condition. Experimental and 
numerical studies yield a critical curve which delineates the capillary number for breakup at each viscosity ratio. With viscoelasticity, however, drop evolution depends on the initial condition. At a given capillary number, the question of whether a drop would break or reach stationary state depends on the initial condition. An abrupt startup induces a drop overshoot which may be sufficiently large to lead to breakup, while a gentle startup may allow the drop to reach a stationary state. A similar scenario is found for viscous flow with the addition of inertia [38]. Experimental results are compared with 3D direct simulations where possible, in order to examine how viscoelastic stresses influence drop dynamics.

\section{Theoretical and experimental methods}

\subsection{Materials}

This section is a brief overview of the materials, the blend systems, and the experimental methods. We refer the reader to $[13,34,6]$ for detailed descriptions. Table 1 summarizes the rheological characteristics of the blend constituents measured in [13]. The viscoelastic material is either a polyisobutylene (PIB) Boger fluid named BF2, or a shear-thinning branched polydimethylsiloxane (PDMS) named BR16. The Boger fluid has a constant shear viscosity $\eta$ and a constant first normal stress coefficient $\Psi_{1}$. Thus, an Oldroyd-B constitutive model is used to describe its rheology, where the solvent viscosity $\eta_{s}$ equals that of the non-volatile solvent (Infineum S1054). The branched PDMS used in system 2 of table 2, displays shear-thinning behavior, and therefore the dynamic data are fitted with an Ellis model. The response of this system is included because it is similar to the Oldroyd-B systems for the shear rates in this paper: $D e_{d}$ is calculated by means of the zero-shear values of the viscosity and first normal stress coefficient. The Newtonian liquids are mixtures of linear PDMS (Rhodorsil) or PIB (Parapol or Infineum). The PDMS mixture, used as the matrix fluid, is saturated with a low molecular weight polyisobutylene (Indopol H50). Table 2 lists the interfacial tension $\Gamma$ and viscosity ratio $\lambda$ of the droplet-matrix systems used in this study. The viscosity ratio is 1.5 in four of them, and 0.75 in the fifth. The NE-NE system is the reference system, containing only Newtonian components. Table 3 tabulates the rheology of BF2 in system 3 for the second-order fluid model useful for small deformation, the single relaxation mode Oldroyd-B model useful for numerical simulation at larger deformation, and a 5-mode Giesekus model [6] which is useful for modeling drop relaxation.

The droplet deformation experiments are performed with a counter rotating plate-plate device based on a Paar Physica MCR 300, as described in [13]. 
Table 1

Rheology of the blend components at experimental conditions. ( ${ }^{a} \eta$ is the zero-shear viscosity; ${ }^{b} \Psi_{1}$ is the zero-shear first normal stress coefficient derived in [6].)

\begin{tabular}{cccccccc}
\hline \multirow{2}{*}{ Polymer } & & Sample & Temp. & $\eta_{p}$ & $\eta_{s}$ & $\eta$ & $\Psi_{1}$ \\
& & & ${ }^{\circ} \mathrm{C}$ & Pa.s & Pa.s & Pa.s & Pa.s \\
\hline \multirow{2}{*}{ PIB } & NE & Parapol 1300 & 25.50 & $\ldots$ & $\ldots$ & 83.5 & $\ldots$ \\
& NE & Infineum mix & 24.45 & $\ldots$ & $\ldots$ & 59.1 & $\ldots$ \\
& VE & BF2 & 26.00 & 12.2 & 25.7 & 37.9 & 212 \\
& & & 26.40 & 11.7 & 24.8 & 36.5 & 197 \\
PDMS & NE & Rhodorsil mix 1 & 26.40 & $\ldots$ & $\ldots$ & 53.8 & $\ldots$ \\
& NE & Rhodorsil mix 2 & 25.50 & $\ldots$ & $\ldots$ & 125 & $\ldots$ \\
& NE & Rhodorsil 30000 & 26.00 & $\ldots$ & $\ldots$ & 28.4 & $\ldots$ \\
& NE & Saturated Rhodorsil & 26.00 & $\ldots$ & $\ldots$ & 25.2 & $\ldots$ \\
& VE & BR16 & 24.45 & $88.6^{a}$ & $\ldots$ & 88.6 & $317^{b}$ \\
\hline
\end{tabular}

Table 2

Blend characteristics at experimental conditions

\begin{tabular}{ccccccc}
\hline & System & Droplet phase & Matrix phase & $\begin{array}{c}\text { Temp. } \\
{\left[{ }^{\circ} \mathrm{C}\right]}\end{array}$ & $\begin{array}{c}\Gamma \\
{[\mathrm{mN} / \mathrm{m}]}\end{array}$ & $\begin{array}{c}\lambda \\
{[-]}\end{array}$ \\
\hline 1 & VE-NE & BF2 & Sat. Rhod. & $26.00 \pm 0.10$ & $2.2 \pm 0.1$ & 1.5 \\
2 & VE-NE & BR16 & Infineum mix & $24.45 \pm 0.03$ & $2.65 \pm 0.05$ & 1.5 \\
3 & NE-VE & Rhodorsil mix 1 & BF2 & $26.40 \pm 0.04$ & $2.0 \pm 0.1$ & 1.5 \\
4 & NE-NE & Rhodorsil mix 2 & Parapol 1300 & $25.50 \pm 0.05$ & $2.7 \pm 0.1$ & 1.5 \\
5 & NE-VE & Rhodorsil 30000 & BF2 & $26.00 \pm 0.10$ & $2.0 \pm 0.1$ & 0.75 \\
\hline
\end{tabular}

The device has two stress controlled motor stages that can be operated independently. Observations can be performed in the velocity-vorticity plane (top view) via a glass prism mounted above the upper plate, while observations in the velocity-velocity gradient plane (side view) are possible through a cup attached to the lower plate. A constant gap spacing of $3 \mathrm{~mm}$ is used. Drops of different sizes are injected into the matrix by means of an in-house injection system. The droplet is deposited near the mid plane between the two plates. Moreover, only droplets with a radius less than $150 \mu \mathrm{m}$ and a radial distance of at least $5 \mathrm{~mm}$ from the edge of the surrounding cup are selected in order to eliminate possible gap effects and deviations from the linear shear flow field [39].

The digital images are analyzed with automated procedures written in the Scion Image software as detailed in $[13,40]$. The droplet shape is fitted to an 


\begin{tabular}{ccc|ccc|c}
\hline \hline \multicolumn{3}{c|}{ Second-order } & \multicolumn{3}{c|}{ Oldroyd-B } & Temp. \\
$\eta$ Pa.s & $\Psi_{1}$ Pa.s $^{2}$ & $-N_{2} / N_{1}$ & $\eta_{s}$ Pa.s & $\eta_{p}$ Pa.s & $\tau$ s & ${ }^{o} \mathrm{C}$ \\
\hline 37.9 & 212 & 0.1 & 25.7 & 12.2 & 8.7 & 26.0 \\
36.5 & 197 & & 24.8 & 11.7 & 8.4 & 26.4 \\
\hline \hline
\end{tabular}

Table 3

\begin{tabular}{c|c|c|c}
\hline \hline $\begin{array}{c}\text { Giesekus } \\
\text { Mode }\end{array}$ & $\tau(\mathrm{s})$ & $\eta_{p}$ (Pa.s) & $\hat{\kappa}$ \\
\hline 1 & 49 & 2.66 & 0.2 \\
2 & 16.9 & 7.43 & 0.00001 \\
3 & 2.03 & 5.82 & 0.00001 \\
4 & 0.187 & 2.69 & 0.2 \\
5 & 0.0131 & 1.39 & 0.2 \\
\hline Solvent & - & 27.2 & - \\
\hline \hline
\end{tabular}

Second-order and Oldroyd-B constitutive models for the sample BF2; cf. [13]. Giesekus model with 5 relaxation modes; cf. Appendix of [6].

equivalent ellipse in a projected plane. In the velocity-vorticity plane $(x-y$ plane), the 'top-view' width $W$ and length $L_{p}$ are measured. The top-view deformation parameter is $D_{p}=\left(L_{p}-W\right) /\left(L_{p}+W\right)$. The side-view or velocityvelocity gradient plane $(x-z$ plane) gives a length $L$ and breadth $B$. These yield the Taylor deformation parameter $D=(L-B) /(L+B)$. The sideview angle of inclination with the flow direction $(x)$ is denoted $\theta[40,13]$. The geometric parameters are non-dimensionalized with respect to the undeformed drop diameter. Close to the critical condition and during the breakup process, the droplet shape deviates significantly from that of an ellipsoid. Under these circumstances, the droplet is analyzed manually by measuring the geometric parameters $L_{p}$ and $W$.

\subsection{Numerical methodology}

The governing equations for each liquid include incompressibility, momentum transport, and the Giesekus constitutive model:

$$
\begin{array}{r}
\nabla \cdot \mathbf{u}=0, \quad \rho\left(\frac{\partial \mathbf{u}}{\partial t}+\mathbf{u} \cdot \nabla \mathbf{u}\right)=\nabla \cdot \underline{\tau}+\mathbf{F} \\
\tau\left(\frac{\partial \mathbf{T}}{\partial t}+(\mathbf{u} \cdot \nabla) \mathbf{T}-(\nabla \mathbf{u}) \mathbf{T}-\mathbf{T}(\nabla \mathbf{u})^{T}\right)+\mathbf{T}+\hat{\kappa} \mathbf{T}^{2} \tau / \eta_{p}
\end{array}
$$




$$
=\eta_{p}\left(\nabla \mathbf{u}+(\nabla \mathbf{u})^{T}\right) \text {. }
$$

The total stress tensor is $\underline{\tau}=-p \mathbf{I}+\mathbf{T}+\eta_{s}\left[\nabla \mathbf{u}+(\nabla \mathbf{u})^{T}\right]$, $\mathbf{T}$ is the extra stress tensor, $\eta_{s}$ is the solvent viscosity, $\eta_{p}$ is the polymer viscosity, $\eta=\eta_{s}+\eta_{p}, \tau$ is the relaxation time. The Giesekus parameter $\hat{\kappa}$ allows for shear-thinning and the physically acceptable range is $0 \leq \hat{\kappa}<0.5 . \hat{\kappa}=0$ yields the OldroydB model. Subscript 'd' will refer to the drop and ' $m$ ' to the matrix liquid. The dimensionless parameters are the viscosity ratio based on total viscosities $\lambda=\eta_{d} / \eta_{m}$, the capillary number $C a=R_{0} \dot{\gamma} \eta_{m} / \Gamma$ where $\dot{\gamma}$ is the shear rate, a Reynolds number based on the matrix liquid $R e=\rho \dot{\gamma} R_{0}^{2} / \eta_{m}$, a Weissenberg number per fluid $W e=\dot{\gamma} \tau$, and a retardation parameter per fluid $\beta=\eta_{s} / \eta$. Alternatively, the Deborah numbers are $D e_{d}=\left(1-\beta_{d}\right) W e /(\lambda C a)$, and $D e_{m}=\left(1-\beta_{m}\right) W e / C a$. The emulsion time scale is $\tau_{e m}=R_{0} \eta_{m} / \Gamma$, and a dimensionless capillary time is $\hat{t}=t \dot{\gamma} / \mathrm{Ca}$. Numerical and experimental results in later sections are presented in terms of the Deborah numbers.

The two in-house volume-of-fluid (VOF) codes used in this paper are described in detail in $[34,41]$, and reconstructs the interface position from the values of a VOF function which represents the volume fraction of one of the fluids in each grid cell. The surface tension force is computed as a body force in the momentum equation, $\Gamma \kappa_{S} \mathbf{n} \delta_{S}$, where $\kappa_{S}$ denotes the mean curvature of the surface, $\mathbf{n}$ is the normal and $\delta_{S}$ is a delta function on the interface. In the continuum limit, $\mathbf{n} \delta_{S}=-\nabla C$ where $C$ is the volume fraction function.

(i) In the Continuum Surface Force formulation (VOF-PLIC-CSF), the derivatives of the VOF function are replaced by finite differences. The interface is reconstructed from the VOF function using the Piecewise Linear Interface Construction scheme. Nonlinear combinations of first and second finite differences of the VOF function are used to calculate the curvature.

(ii) The Paraboloid Representation Of the interface in the Surface Tension force (VOF-PROST) described in [42] is a sharp interface method in which a quadratic surface is optimally fitted to the VOF function for each interface cell and all its neighbors in the least-squares sense. Overall, this code takes about $75 \%$ longer to run than CSF but has more accurate convergence properties; hence, it makes sense to use PROST when CPUs are available, and use CSF when a rough solution would suffice. An example is drop relaxation upon cessation of applied shear [43], in which small deformations are to be resolved spatially over a long time.

The numerical discretization typically involves a computational domain of sides $L_{x}=16 R_{0}, L_{y}=16 R_{0}, L_{z}=8 R_{0}$, and mesh size $\Delta x=\Delta y=$ $\Delta z=R_{0} / 12$, unless stated otherwise. The Reynolds number is 0.05 to 0.1 throughout, to describe the low inertia limit. The timestep is of the order of 
$\Delta t=0.0001 \dot{\gamma}^{-1}$. The code was checked against prior 2D simulations $(-)[20]$. Figure 1 shows side-view drop deformation $D(t) v s$. dimensionless time, at the parameters of fig. $6 \mathrm{a}$ in [20]: $\lambda=1, \dot{\gamma}=1, W e_{m}=0.75, \beta_{m}=0.5, R e=0.05$. The capillary numbers are $C a=0.1,0.2,0.3,0.4,0.5$. The figure also shows that our 3D simulations (-.-) overlap the 2D results for low $\mathrm{Ca}$. Note that the 2D transient deformation displays an overshoot for high capillary numbers but not for low; secondly, the overshoot increases as the capillary number is increased. For $\mathrm{Ca}=0.5$, an undershoot is also observed after the exhibit of the overshoot. The initial transient overshoots in 2D do not appear in the $3 \mathrm{D}$ results, and the $3 \mathrm{D}$ drop deforms significantly more. These results are independent of the mesh size, timestep and Reynolds numbers, demonstrating that the flow is in a low Reynolds number asymptotic limit at $R e=0.05$. For $C a \geq 0.3$, the $2 \mathrm{D}$ results such as overshoots are therefore not an indicator of 3D dynamics. Contours of the trace of the extra stress tensor are displayed in fig. 1 at the stationary states for $C a=0.3$, which is the highest value of the capillary number at which our 3D evolution settles in the figure. At higher capillary numbers, the 2D results settle while the 3D cases are undergoing initial transients. Viscoelastic stresses are higher in the 3D simulation, and the dominant component is the $(1,1)$ component at the drop tips which pull the drop out further. An additional comparison with prior literature was performed for the $3 \mathrm{D}$ calculation in fig. 3 of [10] at $C a=0.3, W e_{m}=1, W e_{m}=2, R e=0.1$. We agree quantitatively with their results on drop deformation vs. time which they obtained with a front-tracking method. 


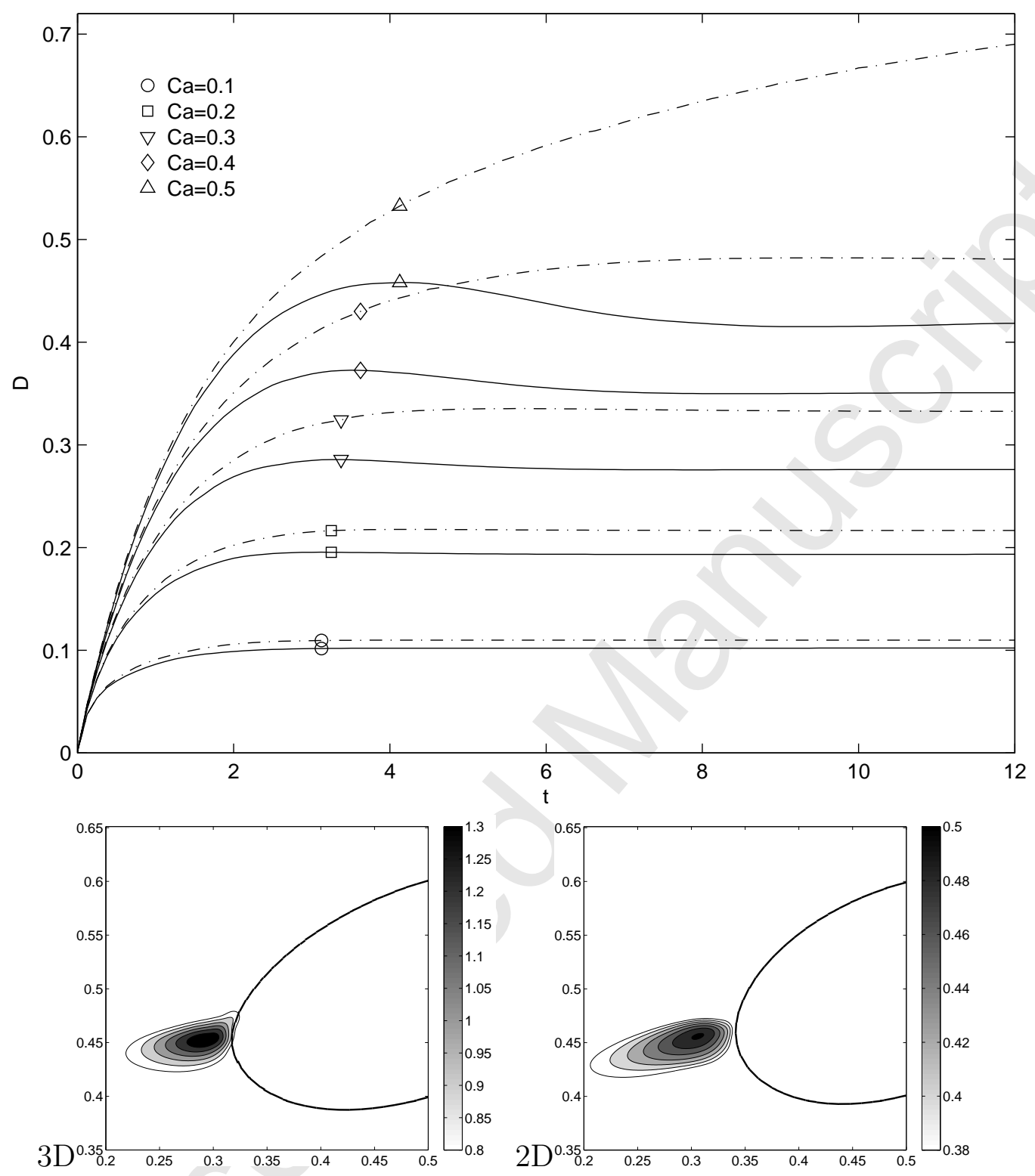

Fig. 1. Newtonian drop in Oldroyd-B matrix. $W e_{m}=0.75, \lambda=1, \dot{\gamma}=1$, drop deformation $D$ vs. $t \dot{\gamma} . C a=0.1,0.2,0.3,0.4,0.5$. VOF-CSF $2 \mathrm{D}$ simulation (-) agrees with fig. 6a of [20], while 3D simulations (-.-) result in higher deformation as $C a$ increases. Contours of the trace of $\mathbf{T}$ at stationary states for $C a=0.3$ in the $x-z$ cross-section are shown for 3D and 2D simulations. 


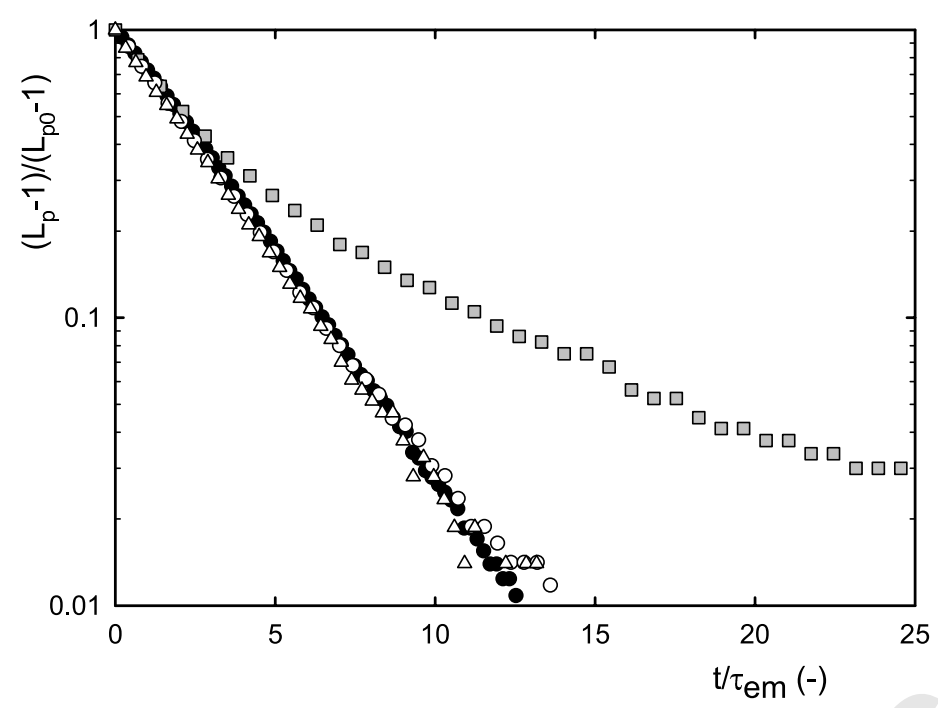

Fig. 2. Shear flow is stopped and droplet retracts to a sphere. $\lambda=1.5$. System 1 , table 2, Boger fluid droplet with $L_{p_{0}}=1.32, C a=0.274, D e_{d}=1.54$ (०). System 2, $L_{p_{0}}=1.24, C a=0.254, D e_{d}=0.77(\triangle)$. System 3, $L_{p_{0}}=1.27, C a=0.361$, $D e_{m}=1.89(\boldsymbol{\square})$. System 4, Newtonian reference system, $L_{p_{0}}=1.31, C a=0.248$ $(\bullet)$.

\section{Drop relaxation upon cessation of shear}

In part 1 of this work, numerical simulations with a single relaxation mode were found to agree quantitatively with experimental data except for the case of a Newtonian drop in the Boger fluid BF2 matrix (system 3, table 2) at higher capillary numbers, which showed long-time decay in deformation that appeared to be inconsistent with a single mode model [6]. This suggested that a multi-mode rheology would be more appropriate at the higher capillary numbers; thus, the 5-mode Giesekus model (table 3), which contains a long relaxation time mode, was developed [6]. Since extending our numerical algorithm to handle more relaxation modes is outside the scope of this paper, one way to check the validity of the multi-mode approach would be to perform relaxation experiments.

Experimental data on drop shape relaxation are taken in the velocity-vorticity plane. Figure 2 compares the viscoelastic systems 1-3 of table 2 with the Newtonian reference system. The evolution of $L_{p}$ is plotted, normalized with respect to its initial value $L_{p_{0}}$. The specific values of $L_{p_{0}}, C a$, and the Deborah number are listed in the figure caption. It is clear that the viscoelastic droplet systems and the Newtonian-Newtonian reference system display the well-known single exponential decay [8, 44]. Thus the influence of droplet viscoelasticity on relaxation is weak, consistent with [19] for weakly viscoelastic droplets. This behavior is found in a wide range of $D e_{d}$ because of the rota- 


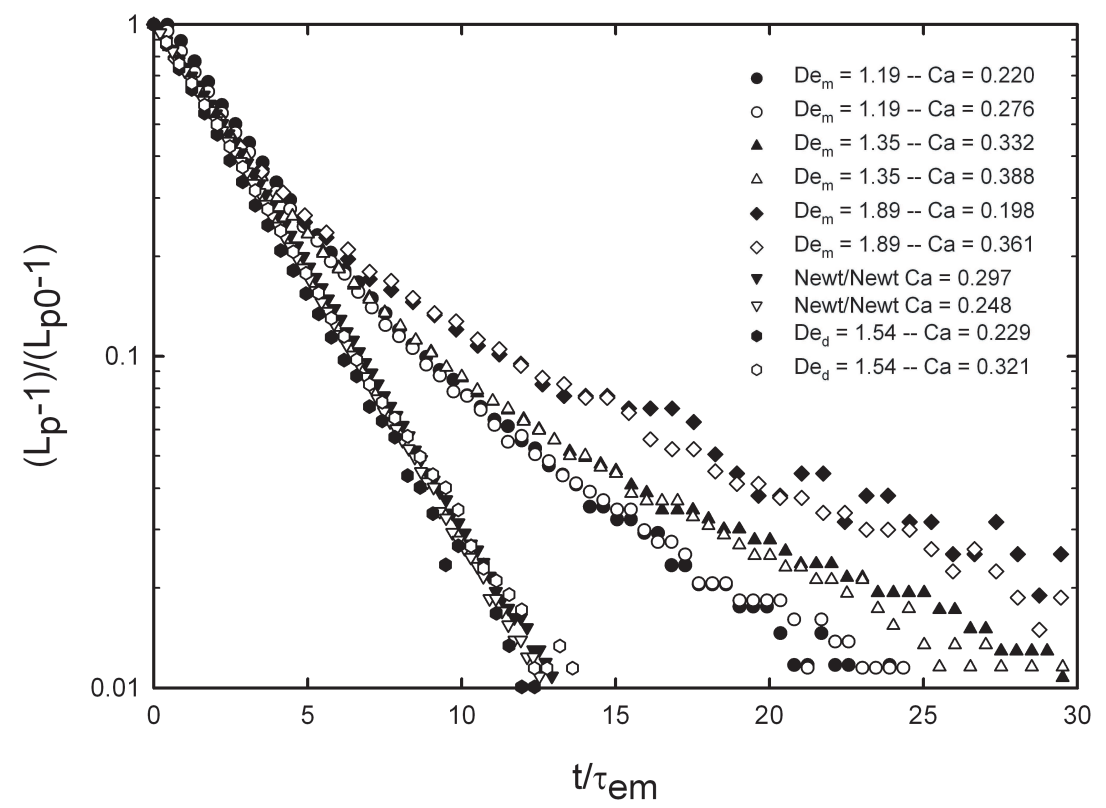

Fig. 3. The figure shows the rates at which droplets retract back to a sphere. $\lambda=1.5$. Capillary and Deborah numbers are given in the legend.

tional flow inside the drop which generates less viscoelastic stresses compared to shear flow.

Matrix viscoelasticity, on the other hand, significantly slows down the relaxation kinetics. This was reported for small initial droplet deformations at viscosity ratio 1 [16] and at viscosity ratio 0.75 [13]. Figure 3 shows that the rate of decay is the same for a given $D e_{m}$, independent of the capillary number; i.e., different initial deformations at fixed Deborah number result in a single master curve for each of the systems we study. Figure 3 shows this for the Boger fluid droplet system 1 and the Boger fluid matrix system 3 of table 2, and the Newtonian-Newtonian system. Moreover, as the Deborah number increases, there is further slowing down of the droplet retraction for the viscoelastic matrix cases.

Figure 4(a) compares the experimental data of the Newtonian reference system with simulations using the PROST method. The figure also shows two numerical results at different spatial refinements $\Delta x=\Delta y=\Delta z=R_{0} / 8$ and $R_{0} / 12$, and shows that the numerical results (- ) converge to the experimental data. The numerical results begin to deviate from the constant slope when the mesh size is comparable to the deviation of the drop to a perfect sphere. The constant decay rate is given by the normal modes of oscillation for a spherical drop. An asymptotic analysis for small-amplitude axisymmetric shape deformations of a viscoelastic liquid drop in vacuum is studied in [37], but an analysis that includes a viscous liquid in place of the vacuum would be required for our 


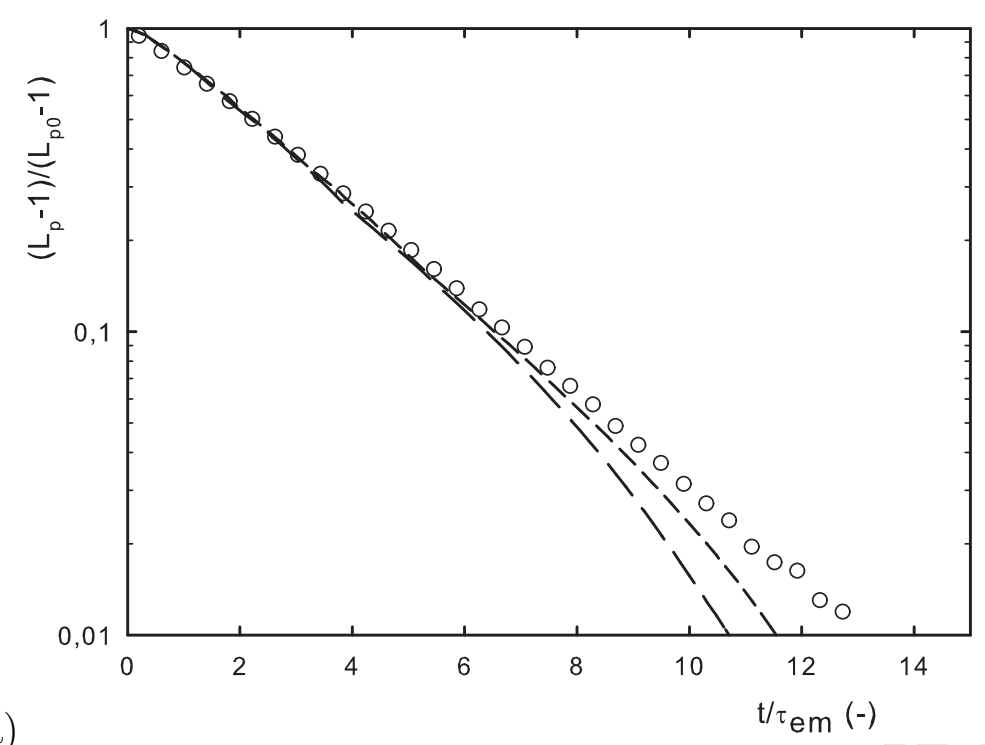

(a)

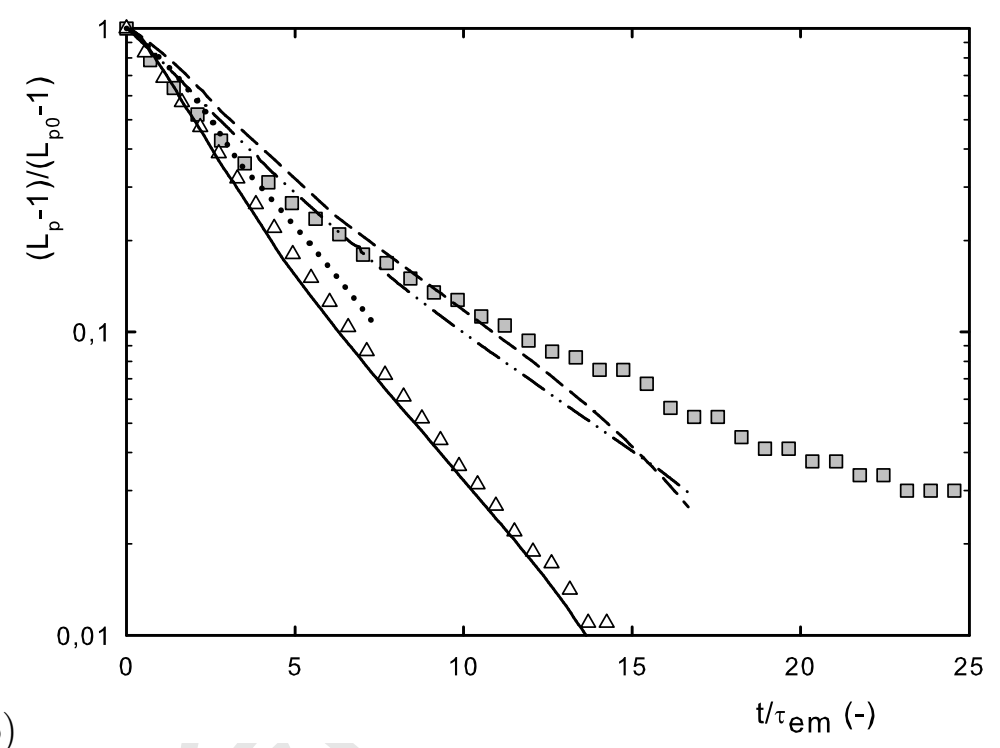

Fig. 4. Experimental data on droplet retraction upon cessation of shear are compared with 3D VOF-PROST simulations. (a) Newtonian-Newtonian reference system, $\lambda=1.5, L_{p_{0}}=1.31, C a=0.248$. Experimental data (o) vs. numerical results with discretizations $\Delta x=\Delta y=\Delta z=R_{0} / 8(--)$ and $R_{0} / 12(--)$. (b) Viscoelastic systems. Boger fluid droplet system at $C a=0.27, D e_{d}=1.02$ : experimental $(\triangle)$ and numerical results with the Oldroyd-B model with grid size $R_{0} / 12(-)$. Boger fluid matrix system with $C a=0.361, D e_{m}=1.89$ : experimental (ם) and numerical $(-.-)$ results. Further numerical results at $C a=0.15, D e_{m}=1.00(\cdots)$ and $C a=0.27, D e_{m}=1.89(--)$.

study.

Figure 4(b) compares the experimental relaxation data with 3D VOF-PROST 
simulations with the Oldroyd-B model for systems 1-3, table 2. Agreement is shown for the viscoelastic droplet system $(\triangle)$. The numerical results for the viscoelastic matrix systems have simple exponential decays related to the relaxation time used in the Oldroyd-B model. The slope at each matrix Deborah number is independent of the capillary number. This is illustrated by the numerical results at $D e_{m}=1.89$ for two different capillary numbers, $C a=0.36$ $(-. .-)$ and $0.27(--)$. Numerical accuracy is of the order of the deviation of the drop from a perfect sphere. An increase in Deborah number $\left(\tau / \tau_{e m}\right)$ increases the difference between the polymer relaxation time and the emulsion time, and slows down the relaxation kinetics. This is evident in the relaxation results for a Newtonian droplet in the Boger fluid matrix: numerical results at $D e_{m}=1(\ldots)$ decay faster than the two results at $D e_{m}=1.89,(-. .-)$ and $(--)$. Viscoelastic stresses at the start of relaxation experiments for $D e_{m}=1$ are concentrated close to the drop tips, and are larger at $D e_{m}=1.89$.

Since the Oldroyd-B model with a single relaxation time results in a simple exponential decay, the experimentally observed change in slope to longer relaxation rates at larger times in figs. 3 and 4(b) would require more modes. The presence of several and longer relaxation times likely causes the change in slope from the initial linear rate. Therefore, a Modified Minale model (Appendix) for a Newtonian drop in a second-order fluid is generalized to handle the 5-mode Giesekus model of table 3. The contribution of each relaxation mode and that of the viscous solvent to the relaxation process is calculated in the following manner. Droplet relaxation is calculated six times with the Modified Minale model; i.e., once for the total viscous contribution, and once for each of the five partial elastic contributions.

For each mode, the matrix fluid is then assumed to behave like a second-order fluid. We choose to calculate the viscous contribution by means of a Newtonian matrix fluid covering the total shear viscosity, while the partial elastic contributions are obtained by using a viscosity ratio of zero and Deborah numbers simply calculated by replacing the relaxation time obtained from the second-order fluid description in table 3 by those of the 5 -mode model. The parameter $-N_{2} / N_{1}$ in the phenomenological model is assumed to be 0.1 for each mode. The final droplet relaxation process is derived from rescaling and adding the contributions:

$$
\left(L_{p}-1\right) /\left(L_{p 0}-1\right)=\sum_{n=0}^{6} S_{n}\left(L_{p, n}-1\right) /\left(L_{p 0, n}-1\right),
$$

where the right hand side sums the different contributions after scaling them with the $S_{n}$ scaling parameter defined by

$$
S_{n}=\frac{\eta_{s}}{\Sigma \eta_{p, n}+\eta_{s}}
$$


for the viscous contribution and

$$
S_{n}=\frac{\eta_{p, n}}{\Sigma \eta_{p, n}+\eta_{s}}
$$

for the partial elastic contributions. Figure 5(a) shows that the experimental data are described quantitatively by this approach. The long tail at the end of the relaxation process is adequately described when the multiple relaxation times of the Boger fluid are taken into account. Moreover, using the same approach, but including the single relaxation time from table 1 yields a droplet relaxation that closely resembles that of the numerical simulation. This is clearly displayed in figure 5(b). Therefore, simulations are expected to yield quantitative agreement with droplet relaxation experiments when multiple relaxation times are implemented in the numerical algorithm, a project that is outside the scope of this work. 


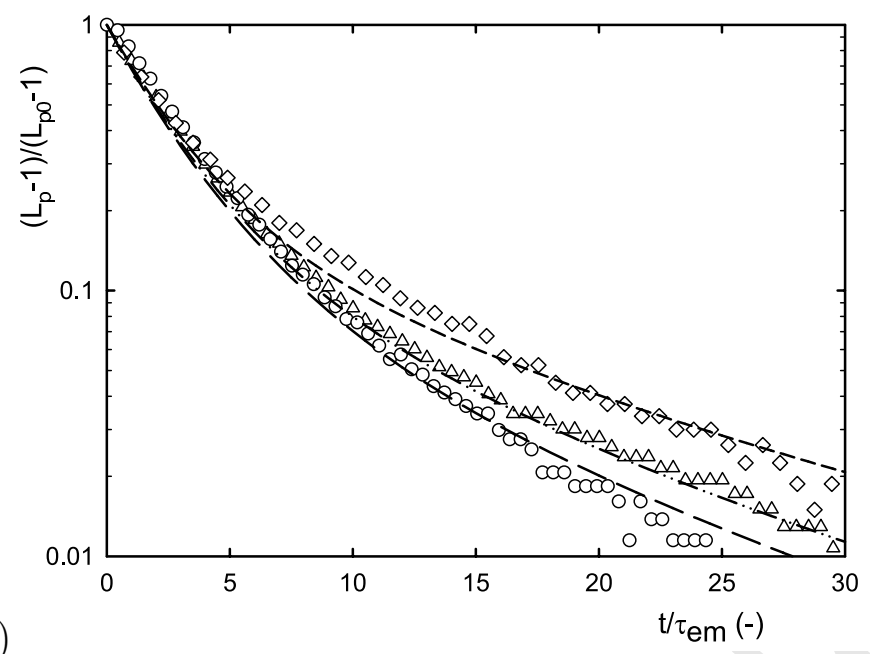

(a)

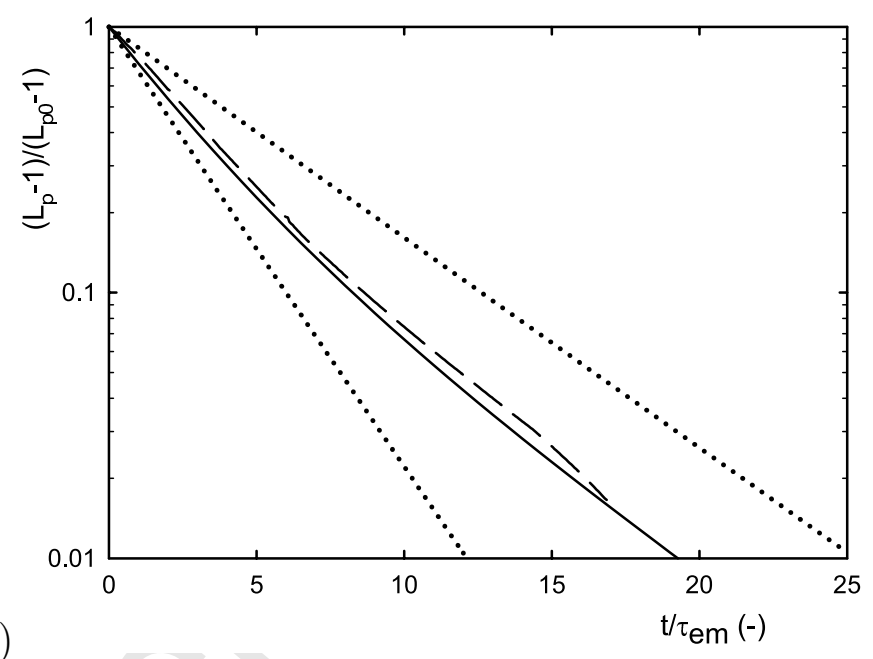

Fig. 5. Droplet retraction upon cessation of shear flow for the Boger fluid matrix system, $\lambda=1.5$. (a) Experimental data are compared with the multi-mode phenomenological model of equations (2) - (4) using the 5 Giesekus modes of table 3. Experimental data at $C a=0.276, D e_{m}=1.19$ ( ○) (- -$) ; C a=0.332, D e_{m}=1.35$ $(\triangle)(-.--) ; C a=0.361, D e_{m}=1.89(\diamond)(--)$. (b) Boger fluid matrix system at $C a=0.361, D e_{m}=1.89$. Comparison between 3D VOF-PROST simulations using Oldroyd-B model (_- ) and the phenomenological model (2) - (4) using the single relaxation time of table $1(-)$. Dotted lines are the unscaled total viscous contribution and the unscaled elastic contribution, calculated separately. 


\section{Drop breakup}

\subsection{Breakup of a Boger fluid droplet in a Newtonian matrix}

An impulsively started shear flow and breakup scenario are studied experimentally for a Boger fluid drop in a Newtonian matrix (system 1, table 2). Figure 6 shows (a) the top-view length $L_{p}$ and $(\mathrm{b})$ width $W$ for a fixed $D e_{d} \approx 1$. Open symbols denote experimental data and lines denote $3 \mathrm{D}$ simulations at a spatial mesh of $R_{0} / 12$. The case $C a=0.27$ shows that the experimental and numerical results are identical. Both VOF-CSF-PLIC and VOF-PROST produced the same results. For $C a>0.41$, a small overshoot in the length becomes evident, and is attributed to the mismatch between the capillary time for drop deformation and the polymer relaxation time of the viscoelastic fluid [20]. Viscous force stretches the drop until viscoelastic stress inside the drop at the tips have time to build up and retract the drop. At $C a=0.5, D e_{d}=0.92$, VOF-PROST is used at mesh size $R_{0} / 12$ and computational box $16 R_{0} \times 16 R_{0} \times 8 R_{0}$ which is sufficient to capture the qualitative features of drop elongation followed by retraction to stationary state. As an aside, this calculation used 16 CPUs (1.5 $\mathrm{GHz}$ speed) on the Virginia Tech SGI Enterprise ALTIX 3700 Supercluster for three days. At $C a=0.57, D e_{d}=0.92$, the droplet undergoes a novel oscillation which results in the first daughter drops conjoined by a filament which eventually pulls them to coalesce, followed by another elongation leading to breakup.

In fig. 6, the initial elongation at $C a=0.57$ is the same for both experimental and numerical results. Figure 7 shows experimental photographs, where the left side is in the velocity-vorticity plane for $C a=0.57$, and the right side is in the velocity-velocity gradient plane at slightly higher $C a=0.59$ and $D e_{d}=$ 1.05. The computed drop shapes at $\hat{t}=9.9$ and 24.7 are close to micrographs 2 and 3. At mesh size $R_{0} / 12$, these numerical results underestimate deformation, but we can study qualitative features of the breakup by simulating at a higher capillary number.

The 3D VOF-PROST simulation in figure 8 at $C a=0.65, D e_{d}=0.92$, qualitatively captures the dynamics in fig. 7 up to the necking regime. The evolution of the topview $L_{p}$ and $W$ are plotted vs. $\hat{t}$ together with the computed side-view shapes. During the early stage, the experimental photos 1-4 in fig. 7 take place during the swift elongation up to $\hat{t} \approx 60$, as in the simulation. Micrographs $5-6$ at $\hat{t} \approx 100$ show necking in the middle of the droplet with the dumbbell ends held together by a thin filament. Interfacial tension pulls each end into ellipsoids which remain connected by the filament. Viscoelastic stresses are shown in the corresponding contours of the trace of the extra stress tensor in fig. 9, which focuses on the filament production. In particular, viscoelastic 


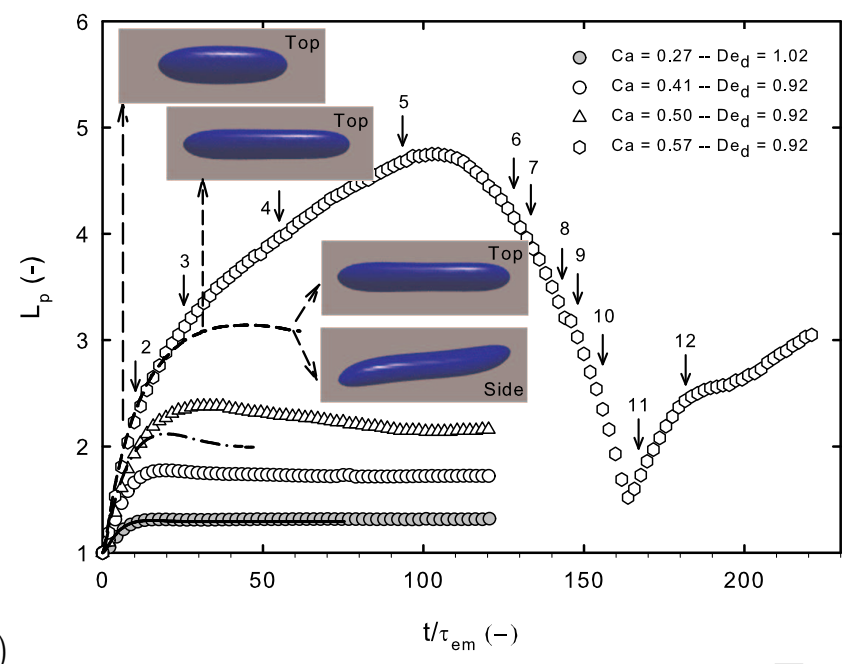

(a)

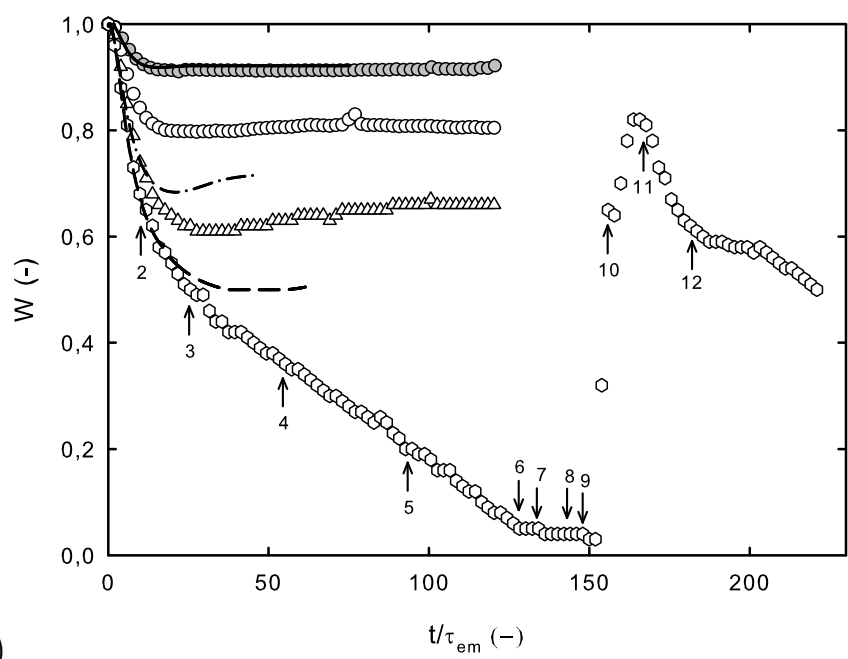

(b)

Fig. 6. Transient droplet deformation and breakup upon startup of shear flow for the Boger fluid droplet, system 1 of table $2 . C a$ is varied for fixed $D e_{d} \approx 1$. The evolution of axis (a) $L_{p}$ and (b) $W$ observed in the velocity-vorticity plane. Experimental data $(\bullet)$ and 3D VOF-CSF simulation $(-)$ at $C a=0.27, D e_{d}=1.02 ; C a=0.41$, $D e_{d}=0.92(\circ) ; C a=0.50, D e_{d}=0.92(\triangle)$, 3D VOF-PROST $(-\cdot-) ; C a=0.57$, $D e_{d}=0.92(\square), 3 \mathrm{D}$ VOF-CSF (--).

stresses initially grow at the drop tip, but when the dumbbell formation begins, they grow many fold in the filament rather than in the satellites. Thus, if the filament were numerically constrained from end-pinching, then it would pull the satellite drops together once a critical stress level is reached. This is consistent with the decrease in $L_{p}$ evident from the 6th photo in fig. 7 . The drop breakup in the numerical method occurs when the size of the filament 


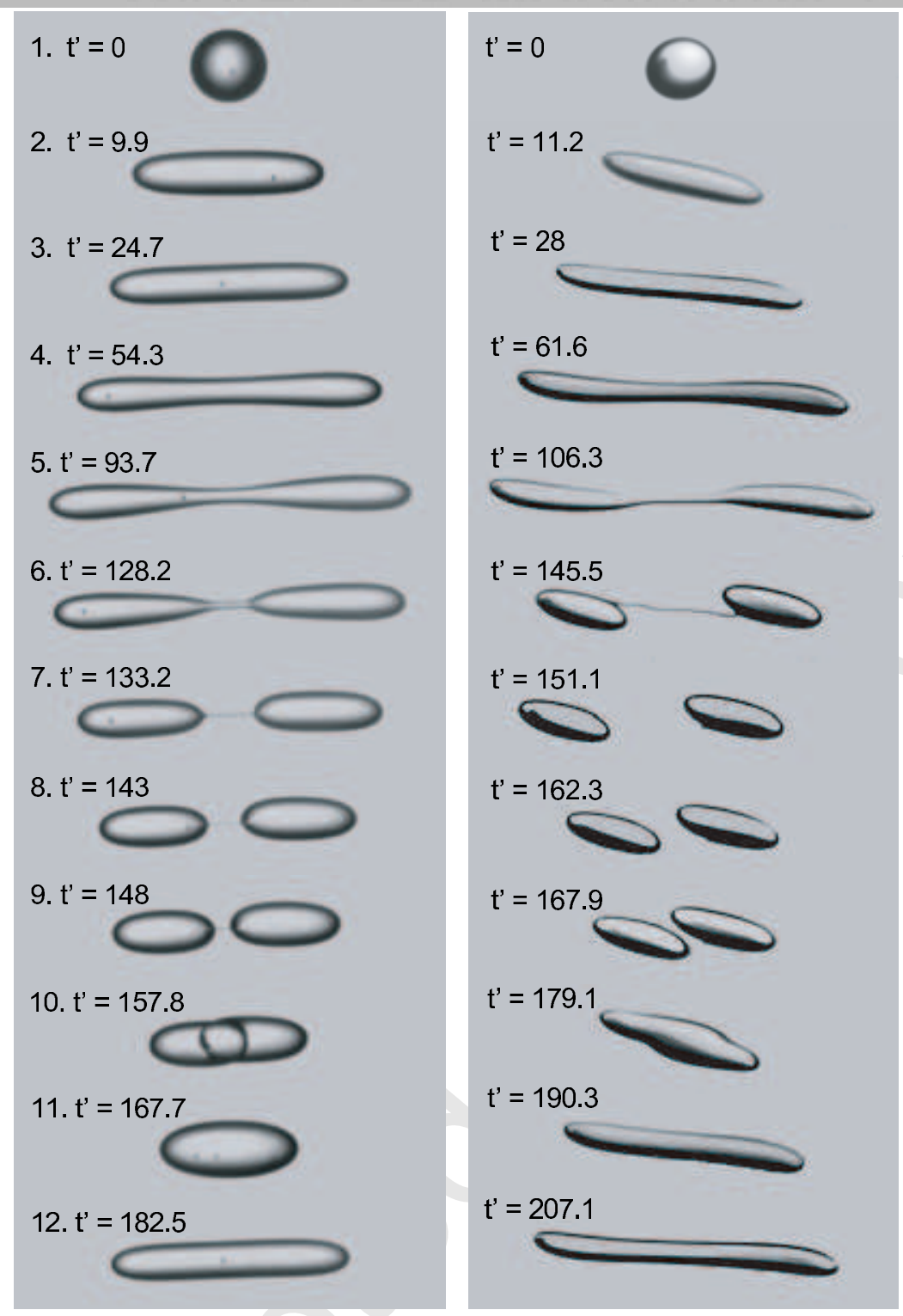

Fig. 7. Micrographs of near-critical behavior for the Boger fluid droplet system, where $t^{\prime}$ denotes $t / \tau_{e m}$ during the first cycle of deformation in figure 6 . Left column: observations in the velocity-vorticity plane, at $C a=0.57$ and $D e_{d}=0.92$. Right column: observations in the velocity-velocity gradient plane at $C a=0.59$ and $D e_{d}=1.05$.

decreases past the grid cell resolution $R_{0} / 12$. Moreover, a true Oldroyd-B filament is likely to continue to thin without breakup. Mathematical analysis on one-dimensional models for surface-tension-driven breakup of a jet in vacuum $[45,46]$ implies that an Oldroyd-B filament never breaks, and that the solution can become beads connected by strings. An important factor is also that the real Boger fluid is expected to deviate from the Oldroyd-B model once the filament becomes extremely thin. 


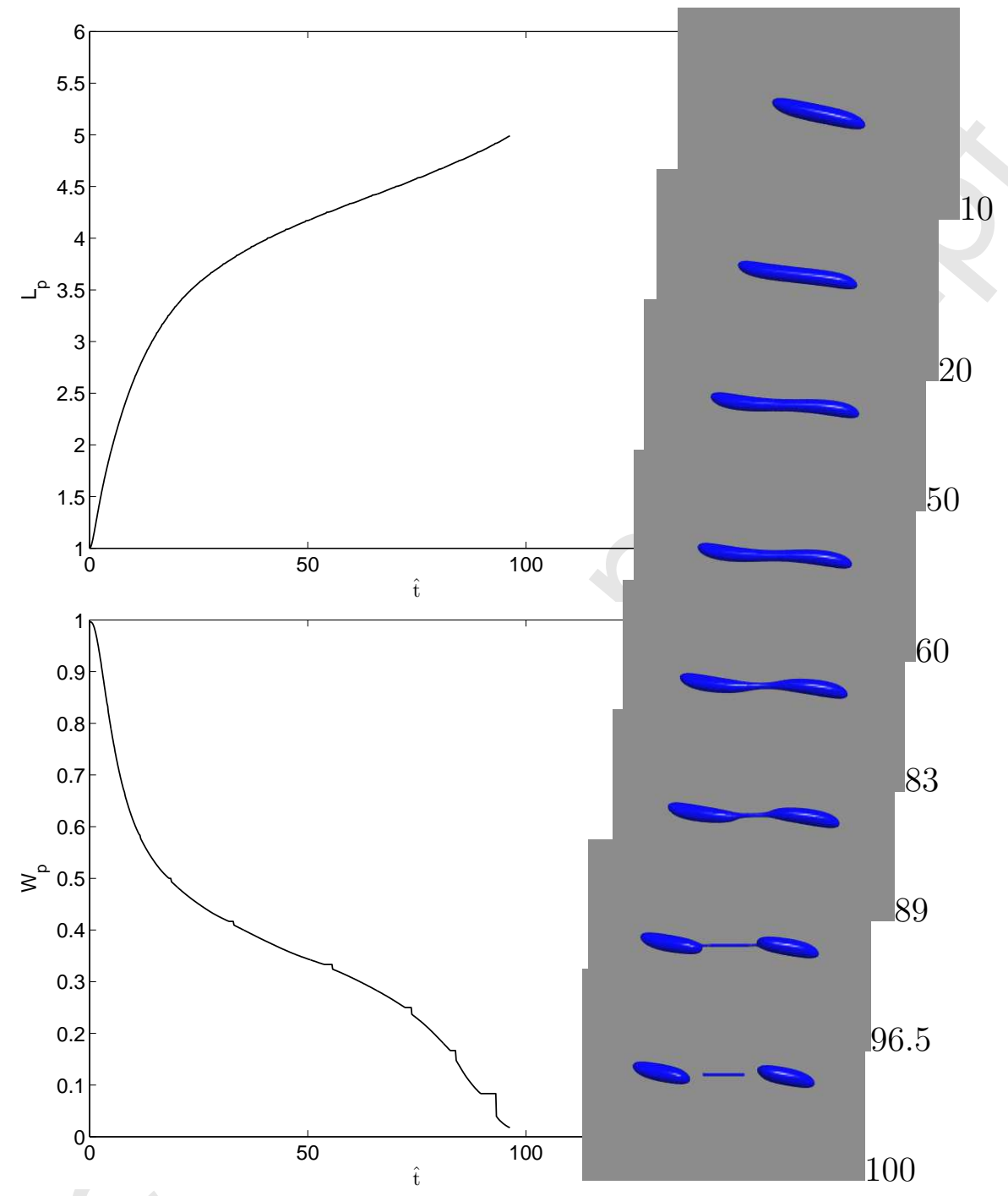

Fig. 8. Numerical results with 3D VOF-PROST, spatial mesh $R_{0} / 12$, timesteps $\hat{t}=0.00025$, at $C a=0.65, D e_{d}=0.92, \lambda=1.5$. The top-view drop length and width are shown against $\hat{t}$. Computational domain $16 R_{0} \times 16 R_{0} \times 8 R_{0}$. Side-view drop shapes are given at $\hat{t}=10,20,50,60,82.6,88.8,96.5,100$. 


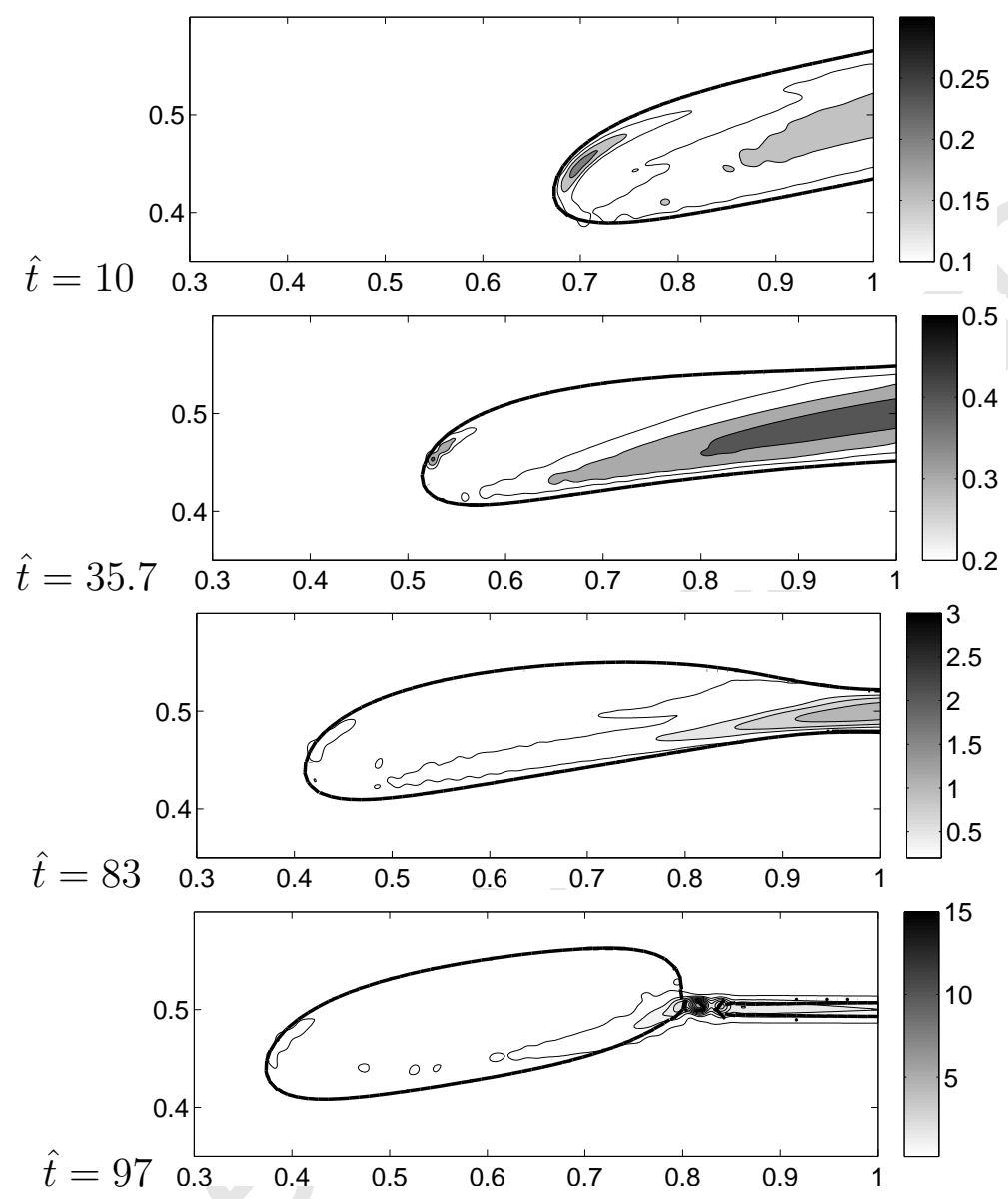

Fig. 9. Contours of the trace of $\mathbf{T}$ corresponding to fig. 8. $\mathrm{Ca}=0.65$, $D e_{d}=0.92, \lambda=1.5 . \hat{t}=10,35.7,83,97$ 
Micrographs 7-9 in fig. 7 show the neck region to have a rather uniform thickness which thins slowly. This is also clear from the plots in figure 6 . An analogous situation is the thinning of a Boger fluid string generated during capillary breakup experiments [47], the common feature being that the dominant flow type within the string is uniaxial flow. The extensional viscosity of our Boger fluid is several decades higher than the shear viscosity (see rheological measurements in the Appendix of [6] and also [48]) and explains the slow thinning of the filament.

Micrograph 10 shows the coalescence after which the drop becomes ellipsoidal. The elastic stresses inside the droplet decrease. Hence, the droplet starts to deform again in micrographs 11 and 12. The second period is not shown in the figure; the main difference is that the neck region grows longer and the string breaks into extremely small satellites. The dimensions of the first two daughter drops are identical, each having roughly half of the volume of the mother drop. Further increase in the capillary number leads to an increase in the length of the initial neck region and coalescence does not occur. The neck typically develops into a beads-on-string formation reminiscent of viscoelastic jets $[49,50]$, together with end-pinching.

\subsection{Breakup of a Newtonian drop in a Boger fluid matrix}

The breakup of a Newtonian droplet in a Boger fluid under simple shear is investigated experimentally in [11] at several viscosity ratios. The end-pinching that takes place for the Newtonian reference system at viscosity ratio roughly 1 is also observed for a highly viscoelastic matrix. The drop deforms gradually without an overshoot. Dumbbells form and become the first daughter drops, held together by a neck that thins until breakup. Two equally sized daughter drops pinch off, with two or more satellite droplets from the neck. Figure 10 shows micrographs for system 5 of table 2 with the Boger fluid matrix at $\lambda=0.75, D e_{m}=1.56$ and $C a=0.50$. This is slightly above the critical capillary number, in agreement with experimental values of $C a_{c r}$ in figure 10 of [11].

Numerical simulations with 3D VOF-PROST (grid size $R_{0} / 12$, computational domain $16 R_{0} \times 16 R_{0} \times 8 R_{0}$ ) are shown below the micrographs, with contour plots of the trace of the extra stress tensor in the velocity-velocity gradient plane. The interface is also shown in the stress plots. The computational domain for half of the drop covers a unit cube, and a portion is shown. The contour for the smallest stress value plotted ( 0.2 on a scale up to 2 for roughly the maximum) can be seen to extend from the left boundary, since periodic boundary conditions are used in the direction of flow, and in the transverse direction. From the beginning, viscoelastic stress is concentrated at the interface 
just outside the drop tip, pulling the ends out. From $t^{\prime}=20$ to 30 , the magnitude of the maximum value increases. The contours that invade into the drop are numerical noise, which appear to be confined through the computation. The stress gradient just outside the interface is significant and the placement of the maximal value appears to shrink with time toward the interface, which adds to the difficulty in numerical resolution. Between $t^{\prime}=60$ and 70 , the drop elongates sufficiently to begin end-pinching, and viscoelastic stress begins to build up at the neck, which may delay breakup. The time to breakup is much longer than in the corresponding Newtonian system. After $t^{\prime}=77$, the daughter drops move away with the flow, while the filament shrinks and breaks due to interfacial tension. Mesh refinement would improve the resolution of the filament and its satellites. 


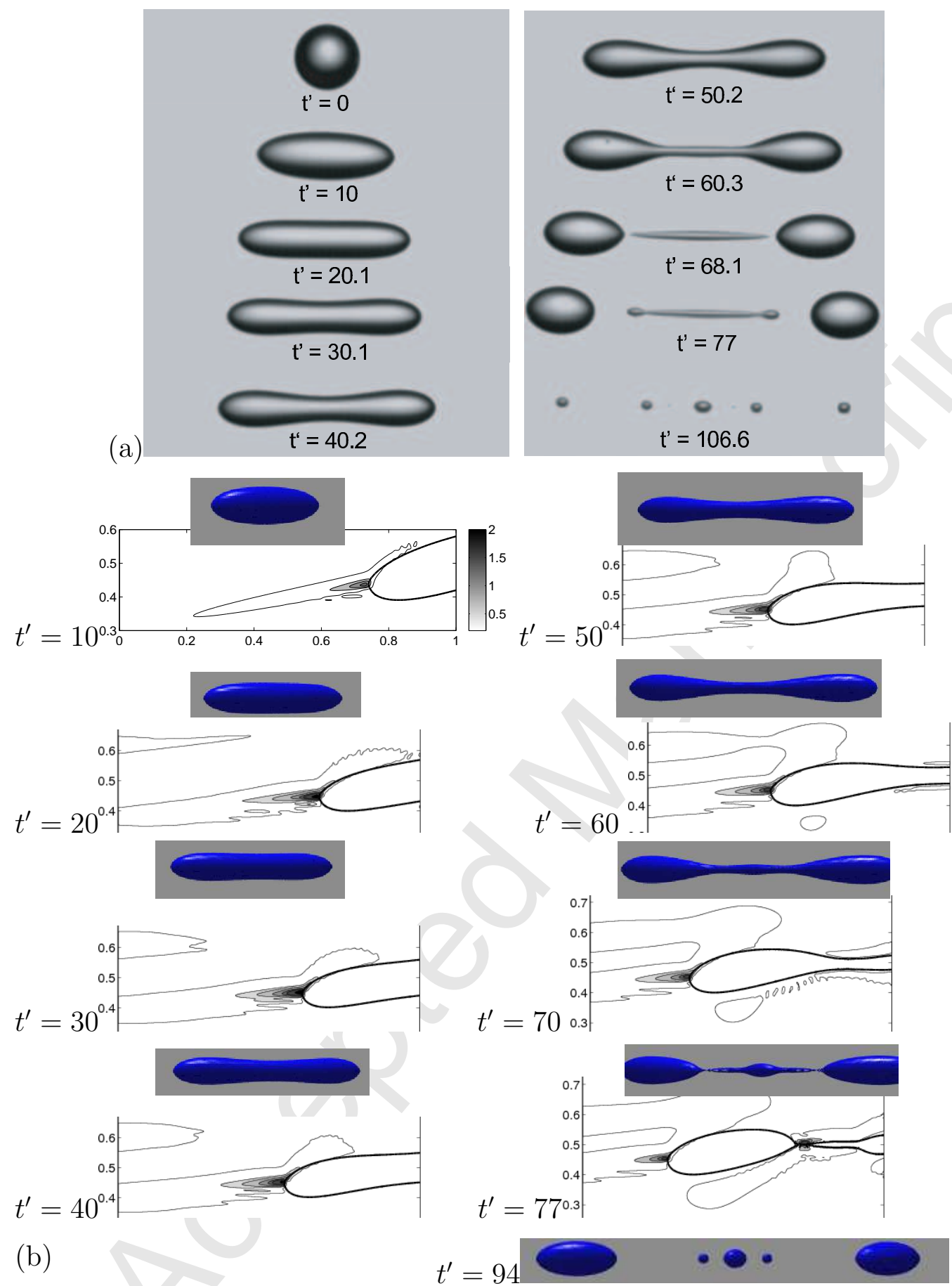

Fig. 10. Breakup of a Newtonian droplet in a Boger fluid matrix (system 5, table 2) observed in the velocity-vorticity plane; $t^{\prime}=\hat{t}=t / \tau_{e m}, C a=0.50, \lambda=0.75$ and $D e_{m}=1.56$. Figure 11 shows that this breakup is prevented when the shear rate is increased in small steps from a lower value. (a) Experimental micrographs. (b) Numerical simulations with 3D VOF-PROST, drop shape and contours of $\operatorname{trace}(\mathbf{T})$. 


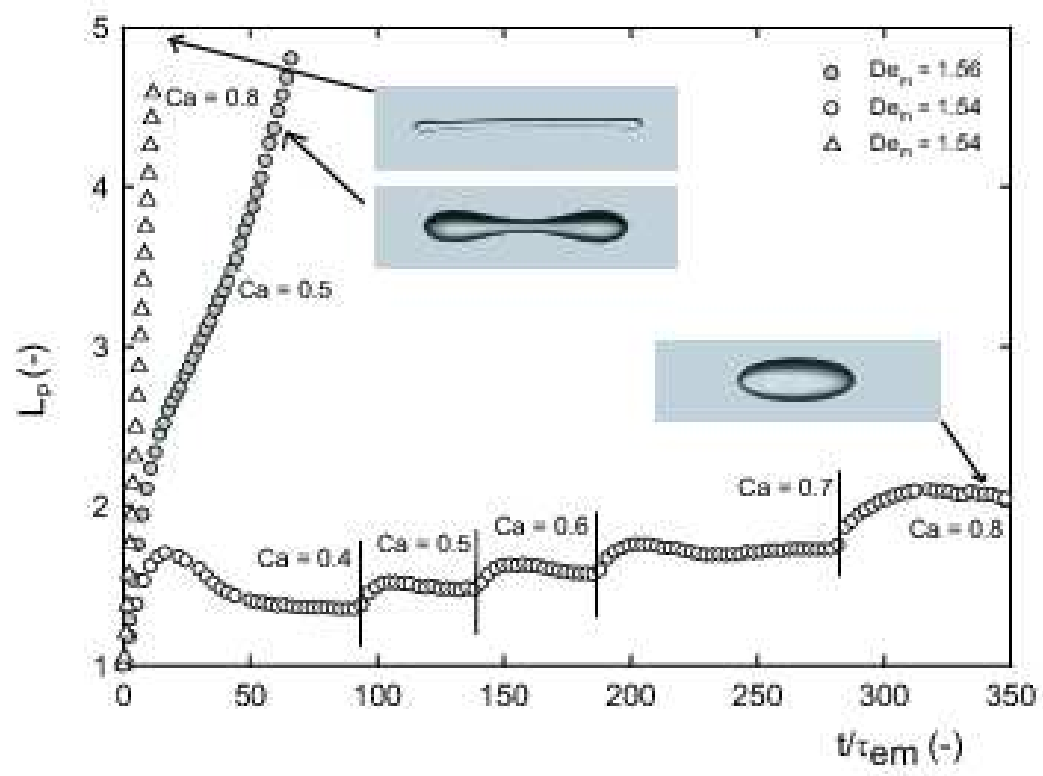

Fig. 11. Experimental data for a Newtonian drop in the Boger fluid matrix (system 5 , table 2) at $D e_{m}=1.54, \lambda=0.75, \beta_{m}=0.68$.

\section{$5 \quad$ Effect of shear flow history}

The uniqueness associated with Stokes flow [38] is lost when viscoelastic liquids are included. A drop may break up if the initial condition is abrupt, or may reach a stationary state if gently ramped to reach the same capillary number. Transient overshoots and oscillations occur naturally in viscoelastic systems close to critical capillary numbers and thus a critical curve is significantly affected by the shear flow history.

Figure 11 shows experimental data for the Newtonian droplet suspended in a Boger fluid (system 5, table 2) at Deborah number 1.54. The major axis $L_{p}$, as observed in the velocity-vorticity plane is plotted against $\hat{t}=t / \tau_{\text {em }}$ for three different experimental runs: a direct startup from rest to $C a=0.8$ at which the slender droplet undergoes a Rayleigh instability and breaks, a direct startup from rest to $C a=0.5$ shown in fig. 10 which end-pinches (gray symbols), and a direct startup from rest to $C a=0.4$ followed by increments of 0.1 up to $C a=0.8$. When started at $C a=0.4$ and stepped up in capillary number, a steady droplet shape results even at $C a=0.8$. The transient overshoots for the incremental step-ups are smaller and may correspond to the mismatch between the capillary time for drop deformation and the polymer relaxation time. Similar experimental results are obtained for viscoelastic droplet systems but omitted for the sake of brevity. We see that the initial overshoot is key to whether the drop breaks or settles. At $C a=0.4$, the overshoot is pronounced but not enough to counteract surface tension, while it is sufficient to continue 


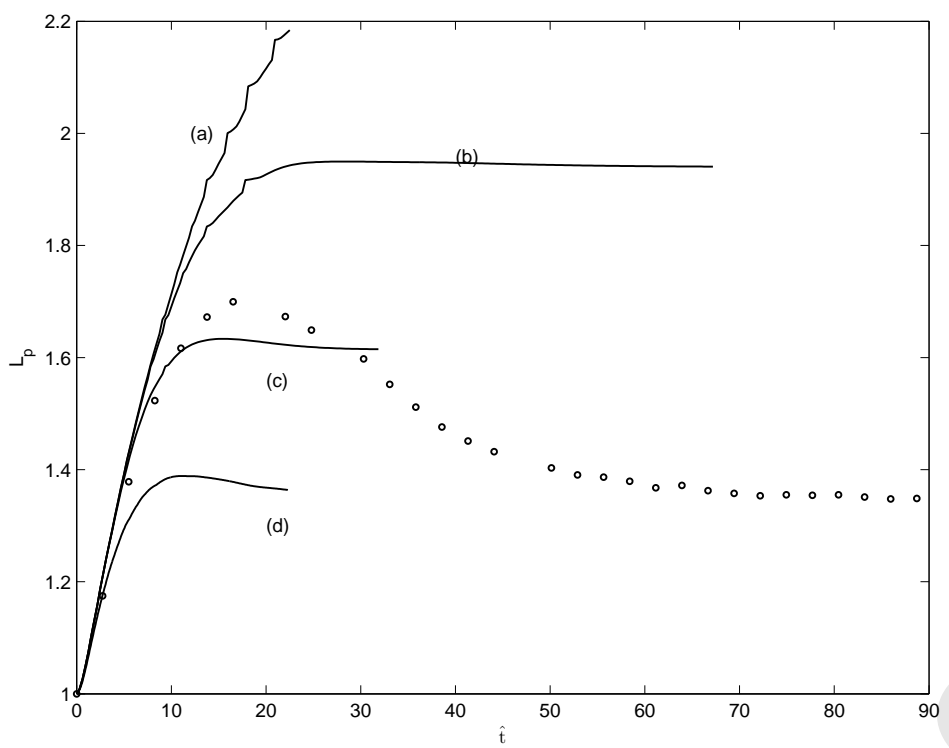

(a) $\hat{t}=20$

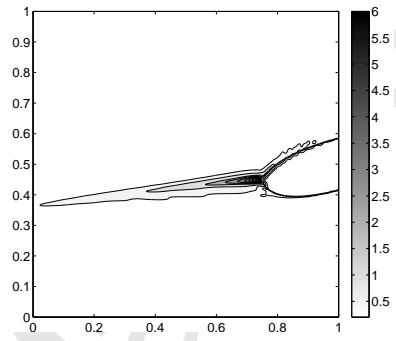

(b) $\hat{t}=60$
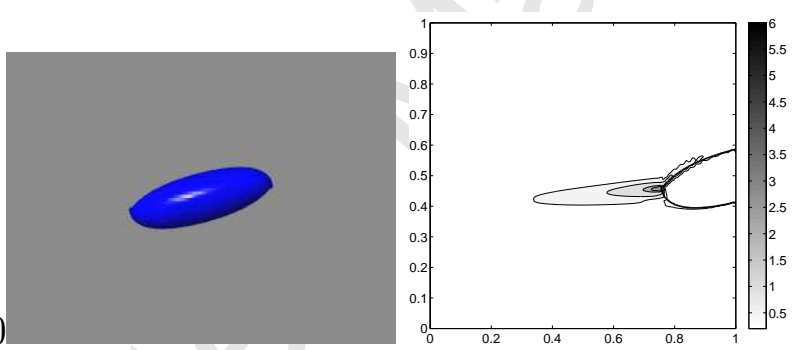

(c) $\hat{t}=30$
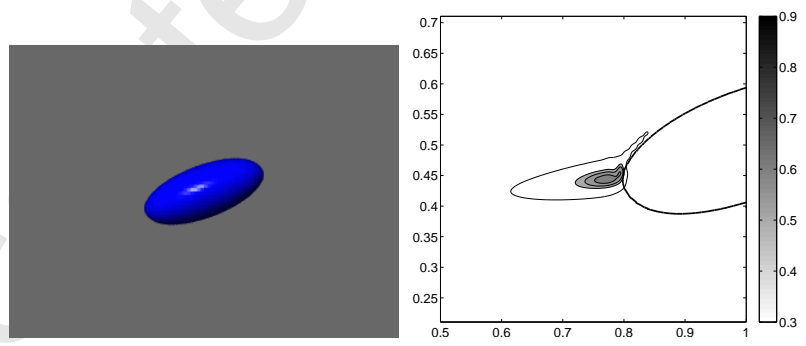

Fig. 12. Numerical results compared with experimental data at the first ramp in figure 11. $C a=0.4, D e_{d}=0, D e_{m}=1.54, \lambda=0.75, \beta_{m}=0.68$. Top-view length $L_{p}$ (o) vs. numerical results of (a) 3D VOF-CSF Oldroyd-B code (-), (b) 3D $\hat{\kappa}_{2}=0.01$, (c) $3 \mathrm{D} \hat{\kappa}_{2}=0.1$, (d) $2 \mathrm{D}$ simulation.

elongation when $\mathrm{Ca}=0.5$. Hence, the history of the flow is important and needs to be characterized well in droplet deformation experiments, making the prediction of droplet behavior in real industrial applications extremely complex. 
Numerical simulations for $C a=0.4, D e_{m}=1.54$, to model the first stepup data in fig. 11 (o) are shown in fig. 12. The 3D VOF-CSF scheme is used with spatial grid size $R_{0} / 12$ in a computational domain of size $16 R_{0} \times 16 R_{0} \times$ $8 R_{0}$. The Oldroyd-B model in (a) develops an instability at the tip where viscoelastic stresses, namely the $(\mathrm{x}, \mathrm{x})$ component, is high in a small region. This area of large gradients in the stresses pulls the drop out. We refined the grid size to $R_{0} / 16$ but the small area of large stress gradients still led to unphysically jutting tips. The development of a cusp at the drop tip may be indicated but the numerical method would not capture that accurately. The extensional rheology of the matrix liquid BF2 is given in the Appendix of [6], showing that the Oldroyd-B model yields a Touton ratio 3 for strain rates $\lesssim 0.05$ and and infinite Trouton ratio for larger strain rates, while the Giesekus model of table 3 yields a Trouton ratio of 3 for strain rates $\lesssim 0.03$, and of order $10^{4}$ for larger strain rates. The measured Trouton ratio for BF2 from strain rates 0.1 to 1 are close to the Giesekus model. The numerical simulation could in principle be use to estimate the elongation rate at the tip, but we require the velocity gradient which is discontinuous at the interface, and therefore difficult to calculate accurately. The high stresses in the numerical simulation with the Oldroyd-B model are an indicator that the flow is approaching the critical elongation rate. The flow would be expected to adjust itself so as not to exceed it. This results in the nibs at the drop tips in (a).

The Giesekus single-mode model with $\hat{\kappa}_{2}=0.01$ is used for fig. $12(\mathrm{~b})$. The drop shape is displayed at $\hat{t}=60$, where the drop tips are nibbed but not as much as in (a). The value of $\hat{\kappa}_{2}$ was increased to 0.1 in (c), resulting in a closer agreement with the experimental initial transient (o) and without the nib development. The stresses at the stationary state have decreased with the increase of $\hat{\kappa}_{2}$ to values in the range of table 3 , and subsequent stepup simulations are can be conducted from (c). A two-dimensional simulation in the velocity-velocity gradient plane is used to generate the velocity-vorticity plane length (d) assuming an ellipsoidal shape. This does not reach the magnitudes in length. In summary, all of the simulations and the experimental data overlap during the initial time period. None of the numerical simulations have the degree of transient overshoot observed in the experiments.

Figure 13 shows 3D VOF-PROST results using the Giesekus model with $\hat{\kappa}_{2}=0.1$ at $D e_{m}=1.54, \lambda=0.75, \beta_{m}=0.68$, to model the experiments of fig. 11. The stepup simulations are started at a much lower capillary number, $C a=0.2$, and increased in smaller steps than the experiments in order to avoid any transient overshoots to reach a stationary state at $C a=0.5$. The startup transient in fig. 10 involves a slight overshoot which is enough to kick the drop to elongate sufficiently for necking to ensue. The stepups provide a different initial condition with the same level of the viscoelastic stresses but in a stationary state. Simulations that do not break up at $C a=0.5$ are found to reach the same stationary state. Numerical simulations for drop deformation 

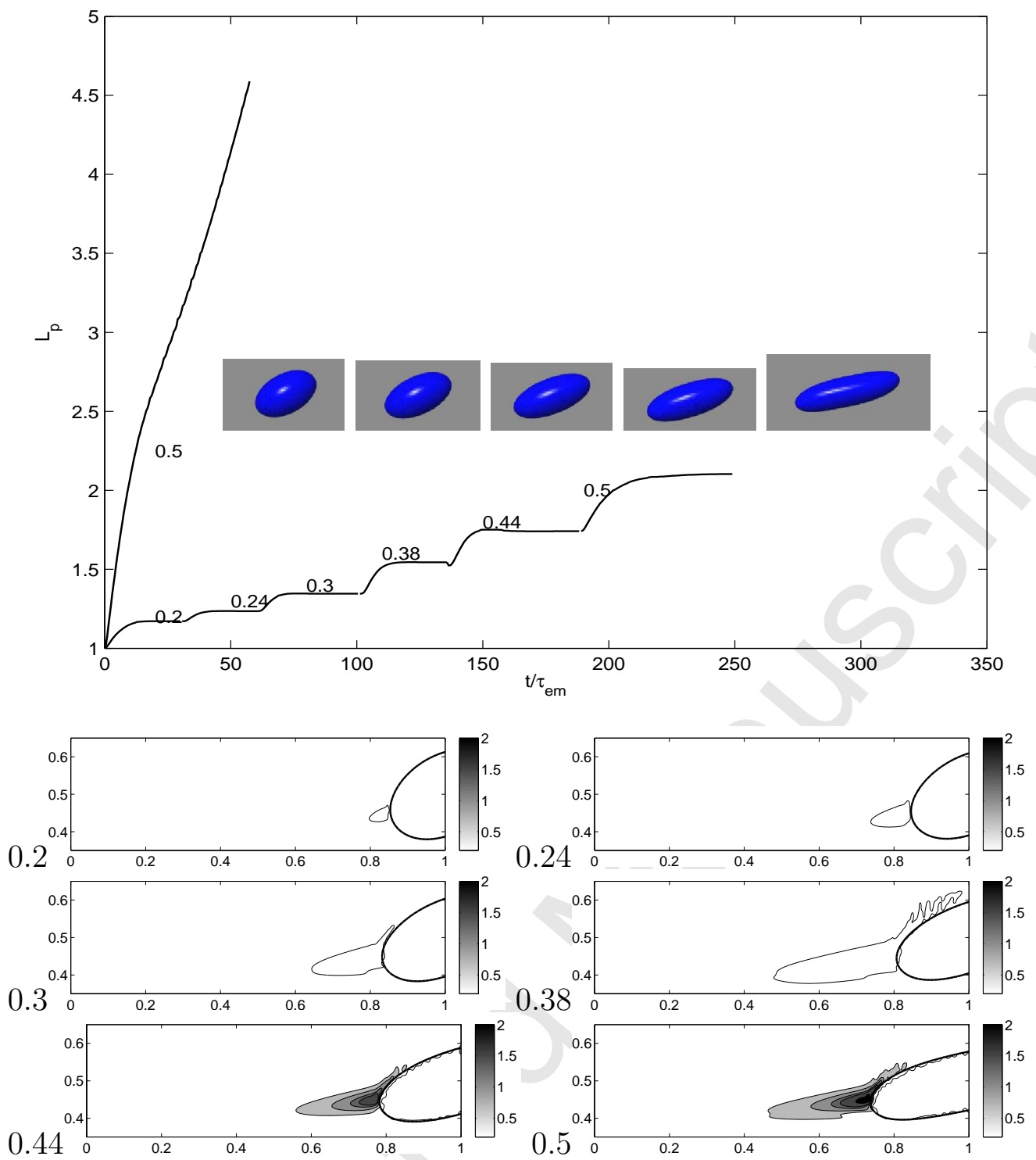

Fig. 13. 3D VOF-PROST with mesh size $R_{0} / 12$ for flow conditions of fig. 11; a Newtonian drop in the Boger fluid matrix (system 5, table 2) at $D e_{m}=1.54$, $\lambda=0.75, \beta_{m}=0.68$. Capillary numbers are displayed in the figure. Droplet shapes at $C a=0.24,0.3,0.38,0.44,0.5$ are superposed. The case which elongates immediately is the startup transient for $C a=0.5$ (cf. fig. 10).

under simple shear typically begin with the drop placed in a device which is started up. For a Newtonian Stokes system, the drop evolves in a monotone manner either to a stationary state or to breakup, independent of the initial condition. When inertia is added, whether a drop breaks up or not again depends on initial conditions [38]. An abrupt startup induces drop oscillations which are sufficiently large to lead to breakup, while a gentle startup can allow the drop to reach a stationary state. 


\section{Conclusions}

The influence of droplet and matrix viscoelasticity on the droplet dynamics is investigated microscopically for blends with a viscosity ratio of 1.5 and 0.75 . In [6], droplet elasticity and shear-thinning behavior were shown to hardly affect the transient droplet deformation and orientation during startup, while the effect of matrix viscoelasticity is more pronounced. For low capillary numbers, the $3 \mathrm{D}$ simulations with the single mode Oldroyd-B model gave quantitative agreement. On the other hand, some features such as long-time behavior of transients for the viscoelastic matrix case at high capillary numbers are not explained. In this paper, we investigate the influence of multiple modes with drop relaxation experiments, which are performed by stopping the shear once a drop reaches approximately a stationary state. Matrix viscoelasticity is found to retard relaxation in comparison with the Newtonian reference system. Numerical simulations with a single mode Oldroyd-B model yield exponential decay, while the experimental data show evidence of multiple modes. We show that the 5-mode Giesekus model from [6], used with the Modified Minale model of [13], adequately predicts the droplet relaxation in a viscoelastic matrix.

A novel breakup mechanism is observed for the viscoelastic droplet. Just above a critical capillary number, a highly stable string is formed between the dumbbells. The viscoelastic string pulls the dumbbells closer prior to pinch-off and even forces them to coalesce, after which the droplet starts to elongate again, leading to a more pronounced string region which breaks before a second coalescence event can occur. Our results for the Boger fluid drop in a Newtonian matrix around $C a=0.6, D e_{d}=1$ is captured by 3D VOF-PROST simulations during the initial stage when the drop is elongating.

Experimental and numerical results are presented for the breakup of a Newtonian drop in a Boger fluid matrix at $D e_{m}=1.54, C a=0.5$ via end-pinching. The effect of shear flow history on transient evolution is discussed for this fluid pair. When the capillary number is ramped up in small steps to $C a=0.5$, a stationary state is reached, while startup at $C a=0.5$ leads to a transient overshoot and breakup. Numerical simulations indicate that the flow at the drop tip may reach the critical strain rate for the Oldroyd-B model. Thus, a Giesekus model is used at $C a=0.4$ and higher in order to capture the qualitative features. The applied shear flow history is therefore extremely important because a gradual increase of the applied shear allows the elastic stresses to build up more slowly, and reach a stationary state, even above the critical condition for an impulsive startup. This complicates the prediction of droplet breakup in viscoelastic systems during industrial processing, where the flow history is complex. 
This research is supported by NSF-DMS-0456086, NCSA CTS060022, and GOA 03/06 and FWO-Vlaanderen for a Ph. D. fellowship for R. Cardinaels. The authors thank Prof. Sridhar (Monash) for measuring the extensional rheology of our samples, and P. Gillioen \& Dr. J. Lauger (Anton Paar) for help with the counter rotating device.

\section{References}

[1] L. A. Utracki. Polymer alloys and blends. Hanser Munich, 1989.

[2] I. Vinckier, P. Moldenaers, and J. Mewis. Relationship between rheology and morphology of model blends in steady shear flow. J. Rheol., 40:613631, 1996.

[3] J. M. Rallison. The deformation of small viscous drops and bubbles in shear flows. Ann. Rev. Fluid Mech., 16:45-66, 1984.

[4] H. A. Stone. Dynamics of drop deformation and breakup in viscous fluids. Ann. Rev. Fluid Mech., 26:65-102, 1994.

[5] P. Dimitrakopoulos. Interfacial dynamics in stokes flow via a threedimensional fully-implicit interfacial spectral boundary element algorithm. J. Comput. Phys., 225:408-426, 2007.

[6] K. Verhulst, R. Cardinaels, P. Moldenaers, Y. Renardy, and S. Afkhami. Influence of viscoelasticity on drop deformation and orientation in shear flow. Part 1: Stationary states. J. Non-Newtonian Fluid Mech., doi:10.1016/j.jnnfm.2008.06.007:., 2008.

[7] P. L. Maffettone and M. Minale. Equations of change for ellipsoidal drops in viscous flow. J. Non-Newtonian Fluid Mech., 78:227-241, 1998.

[8] S. Guido and F. Greco. Dynamics of a liquid drop in a flowing immiscible liquid. In D. M. Binding and K. Walters, editors, Rheology Reviews, pages 99-142. British Society of Rheology, 2004.

[9] N. Aggarwal and K. Sarkar. Deformation and breakup of a viscoelastic drop in a Newtonian matrix under steady shear. J. Fluid Mech., 584:1-21, 2007.

[10] N. Aggarwal and K. Sarkar. Effects of matrix viscoelasticity on viscous and viscoelastic drop deformation in a shear flow. J. Fluid Mech., 601:6384, 2008.

[11] V. Sibillo, M. Simeone, and S. Guido. Break-up of a Newtonian drop in a viscoelastic matrix under simple shear flow. Rheol. Acta, 43:449-456, 2004.

[12] V. Sibillo, S. Guido, F. Greco, and P.L. Maffettone. Single drop dynamics under shearing flow in systems with a viscoelastic phase. In Times of Polymers, Macromolecular Symposiam 228, pages 31-39. Wiley, 2005. 
[13] K. Verhulst, P. Moldenaers, and M. Minale. Drop shape dynamics of a Newtonian drop in a non-Newtonian matrix during transient and steady shear flow. J. Rheol., 51:261-273, 2007.

[14] D. C. Tretheway and L. G. Leal. Deformation and relaxation of Newtonian drops in planar extensional flow of a Boger fluid. J. Non-Newtonian Fluid Mech., 99:81-108, 2001.

[15] P. Yue, J. J. Feng, C. Liu, and J. Shen. Viscoelastic effects on drop deformation in steady shear. J. Fluid Mech., 540:427-437, 2005.

[16] V. Sibillo, M. Simeone, S. Guido, F. Greco, and P.L. Maffettone. Startup and retraction dynamics of a Newtonian drop in a viscoelastic matrix under simple shear flow. J. Non-Newtonian Fluid Mech., 134:27-32, 2006.

[17] P. L. Maffettone, F. Greco, M. Simeone, and S. Guido. Analysis of startup dynamics of a single drop through an ellipsoidal drop model for nonNewtonian fluids. J. Non-Newtonian Fluid Mech., 126:145-151, 2005.

[18] W. Yu, C. Zhou, and M. Bousmina. Theory of morphology evolution in mixtures of viscoelastic immiscible components. J. Rheol., 49:215-236, 2005.

[19] W. Lerdwijitjarud, R. G. Larson, A. Sirivat, and M. J. Solomon. Influence of weak elasticity of dispersed phase on droplet behavior in sheared polybutadiene/poly(dimethyl siloxane) blends. J. Rheol., 47(1):37-58, 2003.

[20] P. Yue, J. J. Feng, C. Liu, and J. Shen. Transient drop deformation upon startup of shear in viscoelastic fluids. Phys. Fluids, 17:123101-123107, 2005.

[21] P. Yue, J. J. Feng, C. Liu, and J. Shen. Diffuse-interfase simulations of drop coalescence and retraction in viscoelastic fluids. J. Non-Newtonian Fluid Mech., 129:163-176, 2005.

[22] F. Gauthier, H. L. Goldsmith, and G. Mason. Particle motions in nonnewtonian media. ii. poiseulle flow. Trans. Soc. Rheol., 15:297-330, 1971.

[23] R. W. Flumerfelt. Drop breakup in simple shear fields of viscoelastic fluids. Ind. Eng. Chem. Fund., 11:312-318, 1972.

[24] R.A. De Bruijn. Deformation and breakup of drops in simple shear flows. PhD thesis, Tech. University of Eindhoven, 1989.

[25] J. J. Elmendorp and R. J. Maalcke. A study on polymer blending microrheology: Part 1. Polym. Eng. Sci., 25:1041-1047, 1985.

[26] P. P. Varanasi, M. E. Ryan, and P. Stroeve. Experimental study on the breakup of model viscoelastic drops in uniform shear flow. Ind. Eng. Chem. Res., 33(7):1858-1866, 1994.

[27] W. J. Milliken and L. G. Leal. A note on the effect of vorticity on the deformation and breakup of polymer drops. J. Non-Newtonian Fluid Mech., 42:231-239, 1992.

[28] K. B. Migler. Drop vorticity alignment in model polymer blends. $J$. Rheol., 44:277-290, 2000.

[29] E. Bartram, H. L. Goldsmith, and S.G. Mason. Particle motions in nonnewtonian media. Rheol. Acta, 14:776-782, 1975. 
[30] L. Levitt, C. W. Macosko, and S. D. Pearson. Influence of normal stress difference on polymer drop deformation. Polym. Eng. Sci., 36:1647-1655, 1996.

[31] F. Mighri and M. A. Huneault. Dispersion visualization of model fluids in a transparent couette flow cell. J. Rheol., 45(3):783-797, 2001.

[32] T. Cherdhirankorn, W. Lerdwijitjarud, A. Sirivat, and R. G. Larson. Dynamics of vorticity stretching and breakup of isolated viscoelastic droplets in an immiscible viscoelastic matrix. Rheol. Acta, 43:246-256, 2004.

[33] F. Mighri and M. A. Huneault. In situ visualisation of drop deformation, erosion, and breakup in high viscosity ratio polymeric systems under high shearing stress conditions. J. Appl. Polym. Sci., 100:2582-2591, 2006.

[34] D. Khismatullin, Y. Renardy, and M. Renardy. Development and implementation of VOF-PROST for 3d viscoelastic liquid-liquid simulations. J. Non-Newtonian Fluid Mech., 140:120-131, 2006.

[35] Y. Renardy. Drop oscillations under simple shear in a highly viscoelastic matrix. Rheol. Acta, 47(1):89-96, 2008.

[36] A. Prosperetti. Normal-mode analysis for the oscillations of a viscous liquid drop in an immiscible liquid. J. de Mecanique, 19:149-182, 1980.

[37] D.B. Khismatullin and A. Nadim. Shape oscillations of a viscoelastic drop. Phys. Rev. E, 63, 2001.

[38] Y. Renardy. Effect of start-up conditions for drop breakup under shear with inertia. Int. J. Multiphase Flow, .:doi : 10.1016 / j. ijmultiphaseflow. 2008. 04.004, 2008.

[39] J. S. Vrentas, D.C. Venerus, and M. Vrestas. An exact analysis of reservoir effects for rotational viscometers. Chem. Eng. Sci, 46:33-37, 1991.

[40] S. Guido and M. Villone. Three-dimensional shape of a drop under simple shear flow. J. Rheol., 42:395-415, 1998.

[41] J. Li, Y. Renardy, and M. Renardy. Numerical simulation of breakup of a viscous drop in simple shear flow through a volume-of-fluid method. Phys. Fluids, 12:269-282, 2000.

[42] Y. Renardy and M. Renardy. PROST: a parabolic reconstruction of surface tension for the volume-of-fluid method. J. Comput. Phys., 183:400421, 2002.

[43] D. Jamet, D. Torres, and J. U. Brackbill. On the theory and computation of surface tension: the elimination of parasitic currents through energy conservation in the second-gradient method. J. Comput. Phys., 182:262276, 2002.

[44] S. Guido and M. Villone. Measurement of interfacial tension by drop retraction analysis. J. Coll. Interf. Sci., 209:247-250, 1999.

[45] M. Renardy. Some comments on the surface-tension driven breakup (or the lack of it) of viscoelastic jets. J. Non-Newtonian Fluid Mech., 51:97107, 1994.

[46] M. Renardy. A numerical study of the asymptotic evolution and breakup of Newtonian and viscoelastic jets. J. Non-Newtonian Fluid Mech., 59:267-282, 1995. 
[47] S.L. Anna and G.H. McKinley. Elasto-capillary thinning and breakup of model elastic liquids. J. Rheol., 45:115-138, 2001.

[48] V. Tirtaatmadja and T. Sridhar. Comparison of constitutive equations for polymer solutions in uniaxial extension. J. Rheol., 39:1133-1160, 1995.

[49] J. Li and M.A. Fontelos. Drop dynamics on the beads-on-string structure for viscoelastic jets: A numerical study. Phys. Fluids, 15:922-937, 2003.

[50] C. Clasen, J. Eggers, M. A. Fontelos, J. Li, and G. H. McKinley. The beads-on-string structure of viscoelastic threads. J. Fluid Mech., 556:283308, 2006.

[51] M. Minale. Deformation of a non-Newtonian ellipsoidal drop in a non-Newtonian matrix: extension of Maffettone-Minale model. J. NonNewtonian Fluid Mech., 123:151-160, 2004.

[52] F. Greco. Drop deformation for non-Newtonian fluids in slow flows. $J$. Non-Newtonian Fluid Mech., 107:111-131, 2002.

\section{A APPENDIX: The Modified Minale Model}

A phenomenological model for the dynamics of a buoyancy-free non-Newtonian drop immersed in a non-Newtonian fluid, subjected to a flow field with a uniform velocity gradient, was developed in [51]. We summarize the derivation here in order to place Eq. (2) in context. The Minale model is based on the assumption that the drop deforms while remaining ellipsoidal and the volume is preserved. The drop shape is described by a symmetric, positive-definite second rank tensor $\mathbf{S}$. The evolution equation is

$$
\begin{aligned}
& \frac{d \mathbf{S}}{d t}=C a(\boldsymbol{\Omega} \cdot \mathbf{S}-\mathbf{S} \cdot \boldsymbol{\Omega}) \\
& -f_{1}(\mathbf{S}-g(\mathbf{S}) \mathbf{I})+C a f_{2}(\mathbf{D} \cdot \mathbf{S}+\mathbf{S} \cdot \mathbf{D}) \\
& +C a f_{3}\left[(\mathbf{D} \cdot \mathbf{S} \cdot \mathbf{S}+\mathbf{S} \cdot \mathbf{S} \cdot \mathbf{D})-(\mathbf{D} \cdot \mathbf{S}+\mathbf{S} \cdot \mathbf{D}) \frac{\mathbf{S}: \mathbf{I}}{3}\right]
\end{aligned}
$$

where the tensors $\mathbf{I}, \mathbf{D}$ and $\boldsymbol{\Omega}$ are the second rank unit tensor, the macroscopic deformation rate tensor and the macroscopic vorticity tensor respectively. The scalar function $g(\mathbf{S})$ satisfies a volume preservation equation,

$$
g(\mathbf{S})=\left(3-2 C a \frac{f_{3}}{f_{1}} I_{\mathbf{S D}}\right) \frac{I I I_{\mathbf{S}}}{I I_{\mathbf{S}}},
$$

where $I_{\mathbf{S D}}, I I_{\mathbf{S}}$ and $I I I_{\mathbf{S}}$ are the first scalar invariant of the tensor $\mathbf{S}: \mathbf{D}$, the second scalar invariant of tensor $\mathbf{S}$, and the determinant of tensor $\mathbf{S}$ respectively. The scalar functions $f_{1}, f_{2}$ and $f_{3}$ are determined in order to recover the asymptotic analytical limits for small deformation where 


$$
\mathbf{S}=\mathbf{I}+C a \mathbf{S}_{\mathbf{1}}+C a^{2} \mathbf{I}_{\mathbf{2}}+O\left(C a^{3}\right) .
$$

At second order, however, the drop deviates slightly from the ellipsoid [52]. Thus, the Minale model recovers Greco's second-order limit for the side-view length $L$, breadth and angle of orientation of the drop but not the top-view width $W$. In addition, the model is required to recover the affine deformation limit, at $\lambda=1$, zero interfacial tension, $D e_{m}=D e_{d}=0$, and the Taylor limit as $\lambda \rightarrow \infty$ for Newtonian systems:

$$
\begin{aligned}
& f_{1}=\frac{120(1+\lambda)(16+19 \lambda)}{q}, \\
& f_{2}=\frac{15(16+19 \lambda)^{2}}{q}+\frac{9 C a^{2}}{4\left(2+6 C a^{2}\right)\left(1+\lambda^{2}\right)} \\
& f_{3}=\frac{q_{1}+4 D e_{m} q_{2}-16 D e_{d} q_{3}}{84(1+\lambda) q}-C_{f_{3}},
\end{aligned}
$$

with

$$
\begin{aligned}
q & =3(3+2 \lambda)(16+19 \lambda)^{2}+8 D e_{m}\left(176+436 \lambda+323 \lambda^{2}\right) \\
& +360 D e_{d} \lambda^{2}(3+2 \lambda) \\
q_{1}= & (16+19 \lambda)\left(880+12849 \lambda+10673 \lambda^{2}\right), \\
q_{2}= & \left(67040+141668 \lambda+114796 \lambda^{2}+30115 \lambda^{3}\right) \\
- & 2 \Upsilon\left(41840+107508 \lambda+105276 \lambda^{2}+32215 \lambda^{3}\right), \\
q_{3}= & \lambda^{2}\left[9(1445+1726 \lambda)-16 \Upsilon_{d}(440+673 \lambda)\right] .
\end{aligned}
$$

$\Upsilon_{d}=-N_{2, d} / N_{1, d}$, where $N_{1}$ and $N_{2}$ are the first and second normal stress difference. The subscripts $\mathrm{d}$ and $\mathrm{m}$ denote the droplet and matrix phase. The term $C_{f_{3}}$ is third order in $C a$. For simple shear flow in the x direction at shear rate 1 , steady-state solutions for the drop semiaxes made dimensionless with initial radius (side-view length $L$, side-view breadth $B$, top-view width $W$ ) and the orientation angle $\theta$ are obtained in closed form[51].

In the Modified Minale model [13], the term $C_{f_{3}}$ is chosen so that $f_{3} \rightarrow 0$ for the affine deformation limit at $\lambda=1$, zero interfacial tension, but without assuming that the Deborah numbers are zero. Hence, $f_{3}$ is replaced by $f_{3}^{*}$ defined by

$$
\begin{aligned}
f_{3}^{*} & =\frac{q_{1}+4 D e_{m} q_{2}-16 D e_{d} q_{3}}{84(1+\lambda) q} \\
- & \frac{20335+6 D e_{m}\left(5613-9106 \Upsilon_{m}\right)-8 D e_{d}\left(1359-848 \Upsilon_{d}\right)}{3675+1496 D e_{m}+360 D e_{d}} \\
& \frac{325 C a}{(1+26 C a)(199+51 \lambda)}
\end{aligned}
$$


The Modified Minale model predicts drop deformation and angle for higher deformations than the original Minale model. 